\title{
CONFLICTS AND COMMON INTERESTS IN COMMITTEES
}

\author{
Li, Hao \\ Sherwin Rosen \\ Wing Suen \\ Working Paper 7158 \\ http://www.nber.org/papers/w7158 \\ NATIONAL BUREAU OF ECONOMIC RESEARCH \\ 1050 Massachusetts Avenue \\ Cambridge, MA 02138 \\ June 1999
}

All opinions expressed are those of the authors and not those of the National Bureau of Economic Research.

(C) 1999 by Li, Hao, Sherwin Rosen, and Wing Suen. All rights reserved. Short sections of text, not to exceed two paragraphs, may be quoted without explicit permission provided that full credit, including (C) notice, is given to the source. 
Conflicts and Common Interests in Committees

Li, Hao, Sherwin Rosen, and Wing Suen

NBER Working Paper No. 7158

June 1999

JEL No. D00, J00

\begin{abstract}
Committees improve decisions by pooling independent information of members, but promote manipulation, obfuscation, and exaggeration of private evidence when members have conflicting preferences. We study how self-interest mediates these conflicting forces. When members' preferences differ, no person ever submits a report that allows perfect inference of his private information. Instead, equilibrium strategies are many-to-one mappings that transform continuous data into ordered ranks: voting procedures are the equilibrium methods of achieving a consensus in committees. Voting necessarily coarsens the transmission of information among members, but is necessary to control conflicts of interest. The degree of coarseness of the equilibrium voting procedure is determined by the extent of conflicting preferences. Though self-interests necessarily reduce the efficient use of information in committees, real information sharing occurs nonetheless. Committees make better decisions on behalf of the "average" (Pareto weighted) member than would any individual on the basis of own information. Committees are viable, though imperfect ways of making decisions when information is dispersed among members.
\end{abstract}

\section{$\mathrm{Li}$, Hao}

University of Hong Kong

School of Economics and Finance

Pokfulum Road

Hong Kong

haoli@econ.hku.hk

Wing Suen

University of Hong Kong

School of Economics and Finance

Pokfulum Road

Hong Kong

hrneswc@hkusua.hku.hk
Sherwin Rosen

Department of Economics

University of Chicago

1126 East 59th St.

Chicago, IL 60637

and NBER

srosen@midway.uchicago.edu 


\section{Introduction}

Small-group decisions are ubiquitous for decisions under uncertainty. Judgment by a jury of one's peers, not by a single person, is the hallmark of the American criminal justice system. Committees recommend hiring and tenure decisions, are essential for project and investment undertakings in business firms, and are used for many administrative decisions in all organizations. Group evaluations bring different points of view to bear on a question. They allow the pooling of information that is not otherwise available to a single decision-maker. But conflict among committee members limits the possibilities for information pooling. It is in the self interest of committee members to manipulate their evidence - to exaggerate favorable data that supports their preferred outcome, or conceal unfavorable data that works against it. This paper studies the tension between information aggregation and strategic manipulation of information in small committee decisions.

The value of aggregating diverse information among group members is an ancient idea. It at least goes back to Condorcet, who proved that voting groups with identical preferences but diverse information make better decisions the larger the group size. The Condorcet Jury Theorem (1785) is a very early application of the law of large numbers, and is further developed by Klevorick, Rothschild and Winship (1984). Only recently have economists and political scientists begun to study how conflicting preferences lead to strategic considerations that reduce the aggregate value of information in real committees. Austen-Smith and Banks (1996) show that Condorcet's theorem

holds true only when members vote "sincerely," as if their evidence alone decides the case. There is a sense in which strategic voting contaminates the scale economies inherent in larger statistical samples. 
The difficulty of eliciting private preferences for public goods in groups has been thoroughly studied (Arrow, 1954 : Gibbard, 1973; Satterthwaite, 1975). The subject of this paper is how small groups make decisions when diverse individual preferences are known to all, but when individuals possess private information that must be elicited in committee deliberations. Only recently has research addressed this issue. See especially the recent papers by Feddersen and Pessendorfer $(1996 ; 1997)$, who developed the role of the pivotal voter in voting games with private information, and the related works on cheap-talk games by Crawford and Sobel (1982) and Austen-Smith (1990). Our work is built on a more natural and familiar information structure relevant for many economic quality control decisions. We show how the likelihood principle is tempered and debased by self-interests of committee members when preferences conflict. Even though the information structure in this model is based on continuously distributed private signals, voting schemes based on rank are the equilibrium outcomes of the committee decision process. The coarsening of cardinal to ordinal information through voting necessarily renders committee decisions imperfect in the first-best sense. Nonetheless, imperfect pooling of information in the committee leads to better decisions than any individual would make based only on own private information.

We consider a committee that must choose between two alternatives. Individual committee members are known to have conflicting interests due to different biases towards the alternatives, but their interests are not diametrically opposed. For example, in a recruitment committee evaluating two candidates in different academic fields, each member may be biased in favor of the job candidate in his own field, but each is willing to choose the other candidate if that candidate is sufficiently better. Conflicting interests 
are compounded by incentives to share information. In the recruitment example, committee members may differ in their perspectives or abilities to evaluate the quality of research in different fields. Since assessments are private, committee decision can depend only on members' reports about their information, not on the actual information itself. This is what causes tension between incentives to manipulate evidence and incentives to share information.

We characterize equilibria of strategic information-reporting for given committee decision rules. The first result is that so long as preferences of individual members are not perfectly aligned, a member will not submit a report that fully reveals his private information. Obfuscation is the rule rather than the exception in committees. Specifically, the equilibrium reporting strategy is a many-to-one mapping from the private information to the report. We analyze the case where equilibrium strategies transform continuous data into ordinal information. Members submit one report if the strength of evidence exceeds a certain personal threshold, and submit another report otherwise. Equilibria with finer partitions that allow for more efficient utilization of private information typically exist, but conflicting interests among committee members impose an upper bound on how fine information partitioning can be. Great conflicts within the committee make fine partitions impossible.

We analyze in detail the two-partition case, which amounts to an equilibrium of strategic voting. The two reports of each member can be interpreted as "yes" (pro) and "no" (con) votes. The voting equilibrium is suboptimal for two reasons. First, information is coarsened and made noisier. Second, the category thresholds are chosen strategically rather than cooperatively. In the recruitment example, anticipating manipulation of evidence by a fellow 
committee member, an individual "exaggerates" evidence that the candidate in his field produces high quality research by voting "yes" to his favored candidate even though he would have voted "no" with the same evidence were all information truthfully revealed. He lowers his own bar because he knows that other members will raise theirs. The incentives for manipulation and counter-manipulation generate an area of disagreement larger than that implied by the inherent conflicts in preferences. They lower the quality of committee decisions. Still, exaggeration is limited, and information is aggregated by the committee, albeit imperfectly. The area of disagreement is bounded from above by the need for members to share their private information. We show that regardless of personal preferences towards the two alternatives, each committee member casts the decisive vote less frequently than if he were to make the decision based on his information only. Moreover, if some committee members are known to have access to more conclusive evidence about the decision, other members will cast their deciding votes less frequently. Better informed members are decisive more often.

We also study how information manipulation and information sharing affect the welfare of committee members. For the committee as a whole, gains from sharing information outweigh distortions from information manipulation. It is never Pareto improving to dissolve the committee and instead take turns to make the decision based on individual evidence only, or to delegate the decision to one member and have him make the decision based on his evidence alone. We show that the ex ante welfare of each individual committee member decreases as the preferences of fellow members diverge further from his. When the preferences of fellow members are sufficiently extreme, the benefits to an individual member of sharing information are outweighed 
by biases and distortions in the committee. He would be better off if he were to dictate the decision.

The strategic voting model is used to address abstention. Although individual committee members always have incentives to influence the outcome of committee decision-making to advance their own interests, the gains from information sharing may be so large that some individual members may find it in their self interest to abstain when their private information turns out to be relatively uninformative. We show that allowing abstention improves the quality of committee decision by reducing strategic manipulations in the committee and allowing information to be used more efficiently.

In the remainder of the paper, the problem of strategic information aggregation is discussed in the classical context of jury decision-making. The language of criminal trials offers a convenient vocabulary that facilitates the exposition. Jurors play no role in acquiring the information presented to them. They have different evidence due to differences in perspectives and capabilities in evaluating the information. For simplicity we assume that the jury consists of two persons. Section 2 establishes that conflicting beliefs and preferences in the committee lead to information garbling. Section 3 studies the strategic voting model, which is a simple equilibrium form of information garbling, in detail. Simultaneous reporting is assumed for most of the paper; equilibrium under sequential reporting is described and compared to simultaneous reporting in terms of strategic commitment. Section 4 extends the analysis to committee decision-making situations where abstention is allowed. Section 5 further generalizes to equilibria of the information game with more partitions and more efficient information aggregation. The analysis in this paper focuses on the tension between conflicting preferences and common interests to share private information for a given committee 
decision rule. In section 6 , we briefly compare committee decision-making under different decision rules.

\section{Manipulation Leads to Garbling}

A verdict of "guilty" or "innocent" must be made by a jury of two persons, $A$ and $B$. Juror $A$ 's prior that the suspect is guilty is $\gamma^{a}$, and the personal costs of type I error of false conviction and type II error of false acquittal are $\lambda_{1}^{a}$ and $\lambda_{2}^{a}$ respectively. Let $k_{1}^{a}=\lambda_{1}^{a}\left(1-\gamma^{a}\right)$ and $k_{2}^{a}=\lambda_{2}^{a} \gamma^{a}$. The ratio $k^{a}=k_{1}^{a} / k_{2}^{a}$ represents the cost of false conviction relative to false acquittal. Juror $A$ also receives an observation (evidence) $Y^{a}=y^{a}$. The distribution of $Y^{a}$ is continuous with density function $f_{i}^{a}(\cdot)$ if the suspect is innocent, or with density function $f_{g}^{a}(\cdot)$ if the suspect is guilty. Parameters and variables for juror $B$ are similarly denoted. We assume that $Y^{a}$ and $Y^{b}$ are independently distributed conditional on guilt or innocence. Conflicts in the jury exist as long as $k^{a} \neq k^{b}$, but interests of jurors are not directly opposed as long as $k^{a}$ and $k^{b}$ are strictly positive and finite. Both care about false conviction and false acquittal. Note that there is no difference in this model between bias as manifested in $\gamma$ and preference as manifested in $\lambda$; only their product matters.

If the vector of signals $\left(y^{a}, y^{b}\right)$ is publicly observable, the optimal committee decision depends on the comparison of conditional expected loss under conviction and under acquittal. Let $\alpha^{a}$ and $\alpha^{b}$ be relative Pareto weights for jurors $A$ and $B$. Define $k_{1}=\alpha^{a} k_{1}^{a}+\alpha^{b} k_{1}^{b}$, and $k_{2}=\alpha^{a} k_{2}^{a}+\alpha^{b} k_{2}^{b}$. It can be 
shown ${ }^{1}$ that conviction is optimal if and only if

$$
\frac{f_{g}^{a}\left(y^{a}\right)}{f_{i}^{a}\left(y^{a}\right)} \frac{f_{g}^{b}\left(y^{b}\right)}{f_{i}^{b}\left(y^{b}\right)} \geq \frac{k_{1}}{k_{2}} .
$$

Throughout this paper, we assume that the signals satisfy a monotone likelihood ratio property. The ratio $f_{g}^{j}(\cdot) / f_{i}^{j}(\cdot)$ is strictly increasing for $j=a, b$. Under this assumption, the optimal decision is monotone in the evidence $y^{a}$ and $y^{b}$.

If $Y^{a}$ and $Y^{b}$ have the same conditional distributions, then for many special distributions, the mean of the signals is a sufficient statistic. In such cases the optimal statistical decision rule (2.1) takes the linear form: "convict if and only if $y^{a}+y^{b} \geq \delta$," where $\delta$ is some pre-determined function of the preference and distribution parameters representing the "standard of proof." More generally, take logarithms of (2.1) and denote the value of the $\log$ likelihood ratios by $\rho^{a}$ and $\rho^{b}$. The optimal rule can be expressed in terms of a linear aggregation of the evidence, namely, convict if and only if $\rho^{a}+\rho^{b} \geq \log \left(k_{1} / k_{2}\right) .^{2}$

The above characterization of the optimal decision rule applies to individual decision-making as well. In particular, if juror $A$ has access to both $Y^{a}$ and $Y^{b}$, then his optimal decision rule is to convict if and only if

$$
\frac{f_{g}^{a}\left(y^{a}\right)}{f_{i}^{a}\left(y^{a}\right)} \frac{f_{g}^{b}\left(y^{b}\right)}{f_{i}^{b}\left(y^{b}\right)} \geq \frac{k_{1}^{a}}{k_{2}^{a}} .
$$

\footnotetext{
1 See, for example, DeGroot (1970). This optimal decision rule is a special case of the Neyman-Pearson lemma.

2 One can think of the "evidence" as the value of the log likelihood ratio instead of the value of the observation itself. In fact, the log likelihood ratio summarizes all the evidence pertinent to the two hypotheses, guilt versus innocence. Under the assumption of conditional independence, linear aggregation of the log likelihood ratio is always the optimal rule. This result holds whether or not $Y^{a}$ and $Y^{b}$ have the same conditional distributions. See, for example, Edwards (1992).
} 
Note that as long as the two jurors differ in preferences or in priors (i.e., $\left.k_{1}^{a} / k_{2}^{a} \neq k_{1}^{b} / k_{2}^{b}\right)$, their personal optimal standards of proof also differ. This is the source of their incentives to misrepresent their own evidence and attempt to tilt the committee decision to their own advantage when signals are not publicly observed.

Jury decisions only can be made on the basis of jurors' reports of their private information. Without loss of generality, suppose that the jury decision rule is to convict if and only if $S\left(r^{a}, r^{b}\right) \geq 0$, where $S(\cdot)$ is strictly increasing in its arguments, and $r^{a}$ and $r^{b}$ are reports of the two jurors on $y^{a}$ and $y^{b}$ respectively. Consider the reporting game where the two jurors report $r^{a}$ and $r^{b}$ simultaneously after learning about their private evidence $y^{a}$ and $y^{b}$. We want to establish that truthful reporting is not an equilibrium strategy as long as the jury decision rule differs from the optimal rule for an individual juror. Let $y^{b}=\phi\left(r^{a}\right)$ be the implicit solution to $S\left(r^{a}, y^{b}\right)=0$, and let $y^{b}=\phi^{a}\left(y^{a}\right)$ be the implicit solution to $S^{a}\left(y^{a}, y^{b}\right)=0$, where $S^{a}$ is the optimal decision function for juror $A$ (that is, if juror $A$ has access to both $Y^{a}$ and $Y^{b}$, his optimal decision rule is to convict if and only if $S^{a}\left(y^{a}, y^{b}\right) \geq 0$.) Suppose juror $B$ always reports his observation $y^{b}$ truthfully. Juror $A$ does not know the value of $B$ 's observation when he submits his report, so he treats $Y^{b}$ as a random variable. If juror $A$ submits report $r^{a}$, the probability of conviction given the jury decision rule is $\operatorname{Pr}\left[S\left(r^{a}, Y^{b}\right) \geq 0\right]=\operatorname{Pr}\left[Y^{b} \geq \phi\left(r^{a}\right)\right]$. On the other hand, conditional on $Y^{a}=y^{a}$, the optimal probability of conviction for this juror is $\operatorname{Pr}\left[S^{a}\left(y^{a}, Y^{b}\right) \geq 0\right]=\operatorname{Pr}\left[Y^{b} \geq \phi^{a}\left(y^{a}\right)\right]$. Thus, juror $A$ can achieve his optimal conviction probability by choosing $r^{a}$ such that $\phi\left(r^{a}\right)=\phi^{a}\left(y^{a}\right)$. Since $\phi$ and $\phi^{a}$ are different functions as long as the decision functions $S$ and $S^{a}$ differ, $r^{a}$ is not equal to $y^{a}$ in general. ${ }^{3}$

\footnotetext{
3 With minor modifications, this results extends to committees with more than two
} 
An example makes the point transparent. Assume that the jurors are asked to report the value of the log likelihood ratio for their private observation, and the jury decision rule is to convict if and only if $r^{a}+r^{b} \geq \log \left(k_{1} / k_{2}\right)$. If juror $B$ reports truthfully $\left(r^{b}=\rho^{b}\right)$, then from (2.1) and (2.2) above, juror $A$ can achieve his first best by reporting

$$
r^{a}=\rho^{a}+\log \left(k_{1} / k_{2}\right)-\log \left(k_{1}^{a} / k_{2}^{a}\right)
$$

Thus, if the cost of conviction for juror $A$ is less than that for the jury $\left(k_{1} / k_{2}>k_{1}^{a} / k_{2}^{a}\right)$, juror $A$ will report a greater likelihood of guilt than is true. Juror $A$ will exaggerate if juror $B$ always tells the truth. ${ }^{4}$

Given the incentive to manipulate reports under any jury decision rule, one may wonder whether there is an equilibrium where each juror takes the other juror's equilibrium manipulation into account when determining his own report. A simple revelation principle argument shows that such a pure strategy equilibrium does not exist. Given any jury decision function, there is no pure-strategy reporting equilibrium where each juror uses an invertible strategy. To see this point, assume that given the decision function $S\left(r^{a}, r^{b}\right)$ there is a reporting equilibrium $\left(R^{a}\left(y^{a}\right), R^{b}\left(y^{b}\right)\right)$ with invertible functions $R^{a}(\cdot)$ and $R^{b}(\cdot)$. Without loss of generality, assume that both reporting functions are strictly increasing. Then reporting truthfully $\left(r^{a}=y^{a}\right.$ and $\left.r^{b}=y^{b}\right)$ is an equilibrium of the reporting game when the decision function is $T\left(r^{a}, r^{b}\right)$, where $T=S\left(R^{a}\left(y^{a}\right), R^{b}\left(y^{b}\right)\right)$. Since $S(\cdot, \cdot)$ is strictly increasing

members. Truthful reporting can be optimal for some members if others report truthfully, but it cannot be optimal for all members unless there is no conflict in the committee.

4 To be sure, exaggeration in the above form of overstatement will not be an equilibrium strategy given rational expectations. The detailed analysis of equilibrium will be presented in the following sections. 
in each argument, and $R^{a}\left(y^{a}\right)$ and $R^{b}\left(y^{b}\right)$ are strictly increasing functions, $T(\cdot, \cdot)$ is strictly increasing in each argument. But we already know that there is no equilibrium where given the decision function $T(\cdot, \cdot)$ each juror reports his observation truthfully, a contradiction. Since invertible strategies allow people to infer the actual observation from the report, the non-existence of equilibrium with invertible strategies demonstrates incentives to garble private information in committee decision making.

Our result that manipulation arising from conflicting interests leads to information garbling is related to Crawford and Sobel's (1982) work on cheap talk games. However, our model studies the problem of strategic information aggregation instead of signaling, and talk in our model is not exactly cheap. We assume the committee decision rule is fixed, and any report submitted by members is fed mechanically into the rule to yield a decision. As a result of this feature, it is not an equilibrium of the game for a committee member to ignore other people's reports and dismiss them simply as cheap talk. To be sure, in our model there are always many "uninformative" equilibria: for example, in the case of unilateral conviction in a two-person jury, both jurors voting for conviction regardless of their private information is an equilibrium. However, these are not the counterpart of the uninformative equilibrium in cheap talk games, because in voting to convict jurors disregard their prior information as well as all the evidence.

\section{Voting as Equilibrium Garbling}

We study the form of information garbling that maps continuous cardinal

data into intervals or ordinal ranks. These "partition equilibria" of the 
information-reporting game restrict information in a natural way that prevents full revelation of private evidence and sustains pure strategies. ${ }^{5}$ For the most part, we focus on the two-partition equilibrium, where each individual's report takes on only two possible values. This is equivalent to voting.

Suppose that the committee decision rule is to convict if and only if $S\left(r^{a}, r^{b}\right) \geq 0$, where $S(\cdot)$ is strictly increasing in its arguments. If $r_{1}^{j}$ is the maximum admissible report and $r_{0}^{j}$ is the minimum admissible report for each juror $j(j=a, b)$, we must have $S\left(r_{1}^{a}, r_{1}^{b}\right) \geq 0$ and $S\left(r_{0}^{a}, r_{0}^{b}\right)<0$. We want to construct an equilibrium where $r_{1}^{a}$ and $r_{0}^{a}$ are the only two reports for $A$ and $r_{1}^{b}$ and $r_{0}^{b}$ are the only two reports for $B$. Suppose that $S\left(r_{1}^{a}, r_{0}^{b}\right) \geq 0$. Then, we must have $S\left(r_{0}^{a}, r_{1}^{b}\right) \geq 0$. Otherwise, the committee decision would depend on $A$ 's evidence only: regardless of juror $B$ 's report, conviction ensues if $A$ submits report $r_{1}^{a}$ (because $S\left(r_{1}^{a}, r_{0}^{b}\right) \geq 0$ and $S\left(r_{1}^{a}, \cdot\right)$ is increasing), and acquittal ensues if $A$ reports $r_{0}^{a}$ (because $S\left(r_{0}^{a}, r_{1}^{b}\right)<0$ and $S\left(r_{0}^{a}, \cdot\right)$ is increasing). The case of $S\left(r_{1}^{a}, r_{0}^{b}\right) \geq 0$ and $S\left(r_{0}^{a}, r_{1}^{b}\right) \geq 0$ may be called "unilateral conviction," because either juror submitting the bigger report $r_{1}^{j}$ ensures conviction, and submitting the smaller report $r_{0}^{j}$ defers the decision to the other juror. If $S\left(r_{1}^{a}, r_{0}^{b}\right)<0$, the opposite happens. For the committee decision to depend on $A^{\prime}$ 's evidence, we must have $S\left(r_{0}^{a}, r_{1}^{b}\right)<0$. This is the case of "unanimous conviction," because submitting a bigger report effectively defers the decision to the other juror while submitting the smaller report ensures acquittal.

\footnotetext{
5 Mixed strategies also prevent full revelation of private information. However, adding independent noises to observed signals cannot be an equilibrium strategy in general, because it is equivalent to a truthful reporting strategy for the combined "evidence" of signal plus noise.
} 
Proposition 3.1. Fix any decision function $S\left(r^{a}, r^{b}\right)$. There exists an equilibrium of the information-reporting game where juror $A$ reports $r_{0}^{a}$ if $y^{a}$ is below some threshold and $r_{1}^{a}$ otherwise, and juror $B$ reports $r_{0}^{b}$ if $y^{b}$ is below some threshold and $r_{1}^{b}$ otherwise.

(i) For the case of unilateral conviction, the thresholds $t_{*}^{a}$ and $t_{*}^{b}$ satisfy

$$
\begin{aligned}
& \frac{f_{g}^{a}\left(t_{*}^{a}\right)}{f_{i}^{a}\left(t_{*}^{a}\right)} \frac{F_{g}^{b}\left(t_{*}^{b}\right)}{F_{i}^{b}\left(t_{*}^{b}\right)}=k^{a}, \\
& \frac{f_{g}^{b}\left(t_{*}^{b}\right)}{f_{i}^{b}\left(t_{*}^{b}\right)} \frac{F_{g}^{a}\left(t_{*}^{a}\right)}{F_{i}^{a}\left(t_{*}^{a}\right)}=k^{b} .
\end{aligned}
$$

(ii) For the case of unanimous conviction, the thresholds $t_{* *}^{a}$ and $t_{* *}^{b}$ satisfy

$$
\begin{aligned}
& \frac{f_{g}^{a}\left(t_{* *}^{a}\right)}{f_{i}^{a}\left(t_{* *}^{a}\right)} \frac{1-F_{g}^{b}\left(t_{* *}^{b}\right)}{1-F_{i}^{b}\left(t_{* *}^{b}\right)}=k^{a}, \\
& \frac{f_{g}^{b}\left(t_{* *}^{b}\right)}{f_{i}^{b}\left(t_{* *}^{b}\right)} \frac{1-F_{g}^{a}\left(t_{* *}^{a}\right)}{1-F_{i}^{a}\left(t_{* *}^{a}\right)}=k^{b} .
\end{aligned}
$$

Proof. Consider only the first case; the second case is similar. Based on the observation $Y^{a}=y^{a}$, juror $A^{\prime}$ 's posterior on the probability that the suspect is guilty is $\eta \gamma^{a} f_{g}^{a}\left(y^{a}\right)$, and the probability that the suspect is innocent is $\eta\left(1-\gamma^{a}\right) f_{i}^{a}\left(y^{a}\right)$, where the normalizing factor $\eta$ is chosen to make the probabilities sum to one. By submitting report $r_{1}^{a}$, juror $A$ ensures conviction. His expected cost (from false conviction) is $\eta k_{1}^{a} f_{i}^{a}\left(y^{a}\right)$. If he submits report $r_{0}^{a}$ instead, the verdict depends on juror $B$ 's report. From $A$ 's point of view, the suspect will be wrongly convicted with probability $1-F_{i}^{b}\left(t_{*}^{b}\right)$, and wrongly acquitted with probability $F_{g}^{b}\left(t_{*}^{b}\right)$. Juror $A$ 's total expected loss from the two types of errors is then $\eta k_{1}^{a} f_{i}^{a}\left(y^{a}\right)\left[1-F_{i}^{b}\left(t_{*}^{b}\right)\right]+\eta k_{2}^{a} f_{g}^{a}\left(y^{a}\right) F_{g}^{b}\left(t_{*}^{b}\right)$. Comparing the costs of the two reports shows that submitting $r_{1}^{a}$ is preferred to submitting $r_{0}^{a}$ if and only if

$$
\frac{f_{g}^{a}\left(y^{a}\right)}{f_{i}^{a}\left(y^{a}\right)} \frac{F_{g}^{b}\left(t_{*}^{b}\right)}{F_{i}^{b}\left(t_{*}^{b}\right)} \geq k^{a} .
$$


The monotone likelihood ratio property implies that reporting $r_{1}^{a}$ is better than reporting $r_{0}^{a}$ for all $y^{a} \geq t_{*}^{a}$. Furthermore, when juror $B$ is using the two-partition strategy, no other report gives a strictly lower cost than does $r_{1}^{a}$ when $y^{a} \geq t_{*}^{a}$. The argument for juror $B$ is symmetric. Q.E.D.

The thresholds for the two members depend only on whether conviction is reached unilaterally or unanimously. For any given decision function $S\left(r^{a}, r^{b}\right)$, one can generally find many values of the reports $\left(r_{0}^{a}, r_{1}^{a}, r_{0}^{b}, r_{1}^{b}\right)$ to support the same equilibrium of threshold reporting strategies. ${ }^{6}$ Similarly, for given values of the reports $\left(r_{0}^{a}, r_{1}^{a}, r_{0}^{b}, r_{1}^{b}\right)$, one can generally find many functions $S\left(r^{a}, r^{b}\right)$ to support the same equilibrium of threshold reporting strategies. In the following analysis of the two-partition equilibrium, we make no reference to the decision function $S\left(r^{a}, r^{b}\right)$, but instead refer to the smaller report of each member as a vote for acquittal and the bigger report as a vote for conviction. The two cases of unilateral conviction and unilateral acquittal give similar results. For brevity of the analysis, we focus on the case of unilateral conviction. Unless otherwise mentioned, the results for unanimous conviction are parallel. The two cases are compared towards the end of this paper.

Notice that Proposition 3.1 derives voting as an equilibrium of information garbling under private information; voting is not assumed to be the committee decision rule. Perhaps an example with an explicit decision function is helpful to illustrate this point. For many distributions where $Y^{a}$ and $Y^{b}$ have the same conditional distributions, the sample mean is a sufficient

6 Given any partition equilibrium, the choice of reports has to be coordinated. The committee may adopt a particular rule to facilitate the coordination. An example is the point system used in sports like figure skating or gymnastics. 
statistic for optimal statistical decision when evidence is public information. The optimal decision rule (2.1) takes the linear form "convict if and only if $y^{a}+y^{b} \geq \delta$," where $\delta$ represents "the standard of proof." Now suppose that evidence is private information and the committee decision function is $S\left(r^{a}, r^{b}\right)=r^{a}+r^{b}-\delta$. To avoid uninteresting indeterminacies that may arise when jurors submit unbounded reports, we assume that reports are restricted to $[0,1]$. Then, a meaningful standard of proof $\delta$ is positive but not greater than 2. When $0<\delta \leq 1$, there is unilateral conviction, and when $1<\delta \leq 2$, there is unanimous conviction. Although the space of permissible reports in the information-reporting game is continuous, the jurors effectively report only "convict" or "acquit" in equilibrium. Furthermore, the standard of proof $\delta$ can vary continuously in the information-reporting game, but in equilibrium, the effective standard of proof can only be requiring at least one vote for conviction $(0<\delta \leq 1)$ or requiring two votes $(1<\delta \leq 2)$.

The equilibrium conditions in the reporting game can be understood in terms of a "pivotal voter" argument (Feddersen and Pesendorfer, 1997). Strategic voting requires that each juror cast his vote as if it were pivotal. For the case of unilateral conviction, juror $A$ 's vote is pivotal if and only if juror $B$ votes for acquittal. The likelihood ratio for the event that $Y^{a}=y^{a}$ and $B$ votes acquittal is given by the left-hand-side of (3.3). The optimal decision rule for juror $A$ is to vote for conviction if and only if this likelihood ratio is greater than or equal to $k^{a}$. An alternative way to see why pivotal voting is optimal is to consider how jurors choose the threshold rule before observing the signal. Anticipating that juror $B$ uses a voting rule with threshold $t_{*}^{b}$, juror $A$ chooses his threshold $t^{a}$ to minimize the expected loss

$$
k_{1}^{a}\left(1-F_{i}^{a}\left(t^{a}\right) F_{i}^{b}\left(t_{*}^{b}\right)\right)+k_{2}^{a} F_{g}^{a}\left(t^{a}\right) F_{g}^{b}\left(t_{*}^{b}\right) .
$$


In the above expression, juror $A$ 's choice of threshold $t^{a}$ affects his expected loss only when $Y^{b}<t_{*}^{b}$. The first order condition for an optimal threshold $t^{a}$ is precisely (3.1).

Information aggregation under strategic voting is analyzed in a series of papers by Feddersen and Pesendorfer $(1996 ; 1997 ; 1998)$. Our model differs from these models of strategic voting in several respects. By using a richer information structure with continuously distributed private signals, we are able to study a richer set of information manipulation in committee decision-making. Instead of the mixed-strategy equilibria of Feddersen and Pesendorfer, we study partition equilibria in the information-reporting game, which will allow us to study in detail obfuscation, exaggeration, and abstention as distinctive forms of evidence manipulation. Also, we do not impose voting as the collective decision rule. We start with an information aggregation rule that is optimal in the absence of strategic manipulation and derive voting as a possible equilibrium outcome of information garbling. In the following analysis of the two-partition equilibrium as an equilibrium of strategic voting, we go beyond the pivotal voting argument of Feddersen and Pesendorfer, which is valid regardless of whether or not the preferences of committee members coincide. We emphasize the role of conflicting interests in committee decision-making, and address how conflicts affect information manipulation and information sharing and the ex ante welfare of the committee members.

\subsection{Uniqueness and stability of equilibrium}

Equations (3.1) define "reaction functions," and the equilibrium is an intersection of the two curves in the $\left(t^{a}, t^{b}\right)$ plane. For $j=a, b$, denote 
$l^{j}(\cdot)=f_{g}^{j}(\cdot) / f_{i}^{j}(\cdot)$, and $L_{*}^{j}(\cdot)=F_{g}^{j}(\cdot) / F_{i}^{j}(\cdot)$. By the monotone likelihood ratio property that $l^{j}$ is an increasing function, $L_{*}^{j}(\cdot)$ is also increasing. Thus, the two reaction functions are downward sloping in the $\left(t^{a}, t^{b}\right)$ plane. A sufficient condition for a unique intersection is that one reaction function is steeper than the other whenever the two intersect. ${ }^{7}$ This condition is satisfied if $l^{j}(\cdot) / L_{*}^{j}(\cdot)$ is monotone. Uniqueness of equilibrium is necessary for analysis of equilibrium properties.

If in addition $l^{j}(\cdot) / L_{*}^{j}(\cdot)$ is increasing, then the equilibrium is globally "stable" in a pseudo-dynamic sense that starting from any initial values the trajectory of the two thresholds converges to the intersection of the reaction curves. A sufficient condition for stability is that juror $A$ 's reaction function is steeper than that of juror $B$. Direct calculations verify that this is true if $l^{j}(\cdot) / L_{*}^{j}(\cdot)$ is increasing. As is the case for many static games, stability in the pseudo-dynamic sense is required to avoid perverse comparative statics (Dixit 1986). Figure 1 depicts the reaction functions for the case where $Y^{a}$ and $Y^{b}$ are normally distributed, which satisfies the increasing condition on $l^{j}(\cdot) / L_{*}^{j}(\cdot)$.

The case of unanimous conviction is analogous. Define $L_{* *}^{j}(\cdot)=[1-$ $\left.F_{g}^{j}(\cdot)\right] /\left[1-F_{i}^{j}(\cdot)\right]$. The monotone likelihood ratio property also implies that $L_{* *}^{j}(\cdot)$ is an increasing function. Therefore, as in the first case, the assumption that $l^{j}(\cdot) / L_{* *}^{j}(\cdot)$ is increasing is sufficient to ensure that equilibrium is unique and stable. For example, if $Y^{j}$ is normally distributed given guilt or innocence, both $l^{j} / L_{*}^{j}$ and $l^{j} / L_{* *}^{j}$ are monotonically increasing.

\footnotetext{
7 Existence of an intersection of two functions can be guaranteed under appropriate assumptions. For example, if for each $j=a, b$ the support of $Y^{j}$ is compact and the likelihood ratio function $l^{j}$ is unbounded over the support, then the Brouwer fixed-point theorem can be applied to the equations (3.1) to show that an equilibrium exists.
} 


\section{Figure 1}

Reaction functions in the two-partition equilibrium

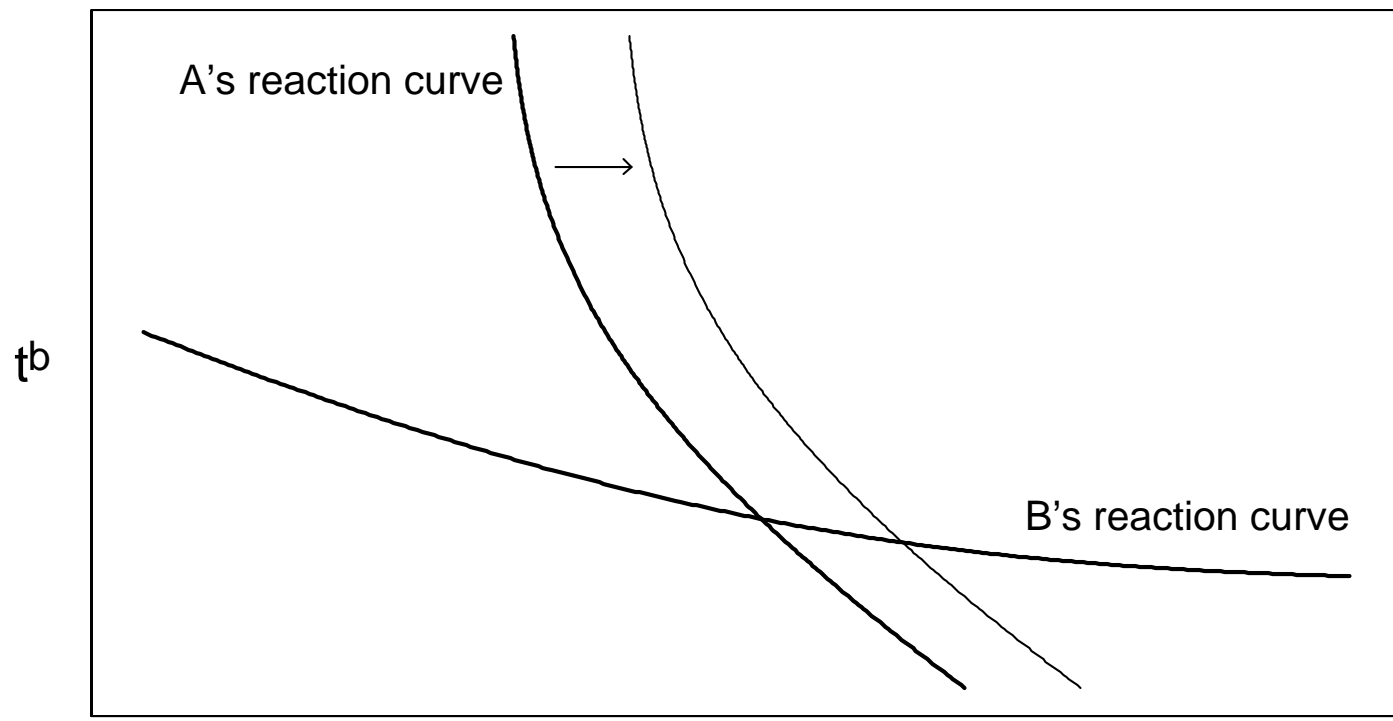

ta

\subsection{Information manipulation and information sharing}

This sub-section presents a few comparative statics results for the information voting game that illustrate the tension between information manipulation and information sharing. With unilateral conviction, equations (3.1) imply that if $Y^{a}$ and $Y^{b}$ have the same conditional distributions, then $k^{a}>k^{b}$ implies $t_{*}^{a}>t_{*}^{b}$. That is, if juror $A$ is more biased toward acquittal than juror $B$, the equilibrium threshold for conviction is higher for juror $A$ than that for juror $B$. For the same observation value $Y^{a}=Y^{b}=y$, juror $A$ votes to acquit while juror $B$ votes to convict if $y \in\left(t_{*}^{b}, t_{*}^{a}\right)$. Therefore $\left|t_{*}^{a}-t_{*}^{b}\right|$ can be thought of as the "area of disagreement" between the two jurors.

The condition that $l^{j} / L_{*}^{j}$ is increasing implies that $d t_{*}^{a} / d k^{a}>0$ and $d t_{*}^{b} / d k^{a}<0$. Thus, the area of disagreement increases as conflict of interests, 
$\left|k^{a}-k^{b}\right|$, increases. As juror $A$ becomes more biased toward acquittal and his standard for conviction increases, juror $B$ counters by lowering his own standard, which induces juror $A$ to increase $t_{*}^{a}$ further. The increase in the equilibrium threshold $t_{*}^{a}$ can be decomposed into two parts: the increase due to shift of $A$ 's reaction function, and the increase along $A$ 's reaction function due to decrease in $B$ 's threshold. See Figure 1. The second part shows that the area of disagreement in committee decision-making is larger than that implied by inherent conflicts in preferences, due to the strategic manipulation and counter-manipulation of reporting thresholds. In this sense conflicts tend to exaggerate favorable evidence. When juror $A$ is more biased toward acquittal than juror $B$, juror $A$ raises his threshold not only because of the concern for false conviction, but also to balance juror $B$ 's opposite tendency to convict. Juror $A$ votes to acquit more often than in the absence of juror $B$ 's manipulation.

Although conflicts cause manipulation, incentives to exaggerate favorable evidence are balanced in equilibrium by incentives to share information. Comparing the equilibrium with how each juror would make the decision based on his own private information shows how jurors share information. If juror $j(j=a, b)$ makes the decision alone, the optimal decision rule convicts if and only if own evidence $y^{j}$ exceeds a threshold $\hat{t}^{j}$ that satisfies:

$$
\frac{f_{g}^{j}\left(\hat{t}^{j}\right)}{f_{i}^{j}\left(\hat{t}^{j}\right)}=k^{j} .
$$

Comparing (3.4) to the equilibrium condition $(3.1)$, since $L_{*}^{j}(\cdot)<1, \hat{t}^{j}$ is lower than $t_{*}^{j}$. When juror $j$ observes evidence $y^{j}$ between $\hat{t}^{j}$ and $t_{*}^{j}$, he votes to acquit even though he would have chosen conviction if he were the only decision-maker. Juror $j$ thus utilizes the information of the other juror 
by casting the decisive vote for conviction less frequently. Note that this is true independent of juror $j$ 's preferences. Even if juror $j$ is biased toward conviction, the need to utilize the fellow juror's information still makes him more "conservative" towards conviction. In the case of unanimous conviction, the decisive vote is acquittal instead of conviction: each juror utilizes the information of the other juror by voting for acquittal less frequently than if the decision were made on the basis of own information.

Incentives to share information under conflicting interests can also be examined by considering how voting behavior changes when one juror's signal becomes more discriminating. To borrow from the concept of statistical power, we say that a signal is more discriminating than another if it results in a lower probability of type I error, holding fixed the probability of type II error. Consider a modification of the structure of information available to the jurors. Juror $A$ still observes $Y^{a}$. Juror $B$ observes $Y^{b}$ with probability $1-\pi$, and observes the true state of guilt or innocence with probability $\pi$. An increase in $\pi$ improves the power of the signal available to juror $B$. The event that $B$ votes acquittal has a likelihood ratio $L_{*}^{b}$, where

$$
L_{*}^{b}=\frac{(1-\pi) F_{g}^{b}\left(t_{*}^{b}\right)}{\pi+(1-\pi) F_{i}^{b}\left(t_{*}^{b}\right)} .
$$

The numerator of $L_{*}^{b}$ is the probability of committing a type II error by juror $B$, and the denominator is one minus the probability of his committing a type I error. Since $L_{*}^{b}$ is decreasing in $\pi$, a higher value of $\pi$ corresponds to more discriminating evidence. In Figure 1 , an increase in $\pi$ causes $A$ 's reaction function to shift to the right. The effect is the same as an increase in $A$ 's bias toward acquittal: $t_{*}^{a}$ increases and $t_{*}^{b}$ decreases. The interpretation is straightforward. Voting to convict decides the verdict regardless of the value 
of the other juror's signal. Voting to acquit, on the other hand, defers the decision to the other juror. When juror $B$ gains access to more discriminating evidence, juror $A$ takes advantage of the improved information by raising $t_{*}^{a}$ and deferring the decision to juror $B$. This is achieved by raising the conviction threshold $t_{*}^{a}$.

The analysis is symmetric for the case of unanimous conviction. Given the modified information structure,

$$
L_{* *}^{b}=\frac{\pi+(1-\pi)\left(1-F_{g}^{b}\left(t_{* *}^{b}\right)\right)}{(1-\pi)\left(1-F_{i}^{b}\left(t_{* *}^{b}\right)\right)} .
$$

An increase in $\pi$ increases $L_{* *}^{b}$, so $t_{* *}^{a}$ falls and $t_{* *}^{b}$ rises. Voting to acquit decides the final outcome of the case. Juror $A$ avoids submitting a decisive vote in order to take advantage of the more discriminating evidence from juror $B$. He therefore lowers $t_{* *}^{a}$ and votes for acquittal less often. Even if juror $A$ is biased toward acquittal, the need to utilize the fellow juror's superior information still makes him more conservative towards casting the decisive vote.

\subsection{Conflicts and welfare}

Conflicts reduce the ex ante welfare of jurors. There is a close relation between the extent of divergence in preferences, $\left|k^{a}-k^{b}\right|$, and expected losses in the information voting game. With unilateral conviction, equilibrium expected loss to juror $A$ is given by

$$
\mathrm{E}\left[C^{a}\left(Y^{a}, Y^{b}\right)\right]=k_{1}^{a}\left(1-F_{i}^{a}\left(t_{*}^{a}\right) F_{i}^{b}\left(t_{*}^{b}\right)\right)+k_{2}^{a} F_{g}^{a}\left(t_{*}^{a}\right) F_{g}^{b}\left(t_{*}^{b}\right) .
$$

Differentiating with respect to $k^{b}$ and using the equilibrium condition (3.1) for juror $A$,

$$
\frac{d \mathrm{E}\left[C^{a}\left(Y^{a}, Y^{b}\right)\right]}{d k^{b}}=\left[-k_{1}^{a} f_{i}^{b}\left(t_{*}^{b}\right) F_{i}^{a}\left(t_{*}^{a}\right)+k_{2}^{a} f_{g}^{b}\left(t_{*}^{b}\right) F_{g}^{a}\left(t_{*}^{a}\right)\right] \frac{d t_{*}^{b}}{d k^{b}} .
$$


Since $d t_{*}^{b} / d k^{b}>0$ when $l^{j} / L_{*}^{j}$ is increasing, $d \mathrm{E}\left[C^{a}\left(Y^{a}, Y^{b}\right)\right] / d k^{b}$ has the same sign as $k^{b}-k^{a}$. For example, if $k^{b}>k^{a}$, a further increase in $k^{b}$ raises juror A's expected loss in the equilibrium.

Conflicts reduce welfare because strategic manipulation becomes more important. A committee with less cooperation uses information less efficiently and welfare in the voting game is lower than in a full information equilibrium. Equation (3.5) shows that $d \mathrm{E}\left[C^{a}\left(Y^{a}, Y^{b}\right)\right] / d k^{b}$ has the same sign as $k^{b}-k^{a}$, and similarly $d \mathrm{E}\left[C^{b}\left(Y^{a}, Y^{b}\right)\right] / d k^{a}$ has the same sign as $k^{a}-k^{b}$. If $k^{a}=k^{b}$ there is no conflict of interest in the jury, and equilibrium threshold choices minimize the expected loss for both jurors. If $k^{a}<k^{b}$, raising the equilibrium threshold for juror $A$ and lowering it for juror $B$ will reduce the expected loss for both jurors, because the first order gain will outweigh the second order loss. If we define "cooperative decision-making" as choosing" thresholds to minimize a weighted sum of expected loss for the two jurors, then conflicts in preferences generate incentives to deviate from the cooperative decision-making. Starting from the cooperative solution, if juror $A$ is more concerned with false acquittal than juror $B$ is, $A$ will lower his threshold for conviction, which induces $B$ to raise his threshold in order to balance $A$ 's bias for conviction. In equilibrium, both jurors are made worse off.

\subsection{Do-it-yourself, delegation, and taking turns}

Since conflicting preferences lead to strategic manipulation in voting and welfare loss for both jurors, do the gains from information sharing in a committee will be sufficient to outweigh the losses from strategic voting? In general the answer is "yes." Even though votes are manipulated, aggregation produces better outcomes than alternatives that do not aggregate private information when conflicts are not too large. 
Let $\mathrm{E}\left[C^{a}\left(Y^{a}\right)\right]$ denote juror $A$ 's unconditional expected loss when he alone makes the decision based on his own information. Then

$$
\mathrm{E}\left[C^{a}\left(Y^{a}\right)\right]=k_{1}^{a}\left(1-F_{i}^{a}\left(\hat{t}^{a}\right)\right)+k_{2}^{a} F_{g}^{a}\left(\hat{t}^{a}\right),
$$

where the optimal threshold $\hat{t}^{a}$ satisfies condition (3.4). Consider the difference $D_{1}^{a}=\mathrm{E}\left[C^{a}\left(Y^{a}, Y^{b}\right)\right]-\mathrm{E}\left[C^{a}\left(Y^{a}\right)\right]$ as a function of $k^{b}$. We showed above that $d \mathrm{E}\left[C^{a}\left(Y^{a}, Y^{b}\right)\right] / d k^{b}<0$ for $k^{b}<k^{a}$ and $d \mathrm{E}\left[C^{a}\left(Y^{a}, Y^{b}\right)\right] / d k^{b}>0$ for $k^{b}>k^{a}$. Since $\mathrm{E}\left[C^{a}\left(Y^{a}\right)\right]$ is independent of $k^{b}$, the difference $D_{1}^{a}$ decreases for $k^{b}<k^{a}$ and then increases for $k^{b}>k^{a}$, reaching a minimum at $k^{b}=k^{a}$. In the limiting case when $k^{b}$ approaches infinity, juror $B$ always votes acquittal and lets juror $A$ make the decision. Therefore, $D_{1}^{a}=0$. At the other limit, when $k^{b}$ approaches zero, juror $B$ ensures conviction by himself. Juror $A$ 's expected loss is then simply $k_{1}^{a}$, and the difference $D_{1}^{a}$ is given by

$$
k_{1}^{a} F_{i}^{a}\left(\hat{t}^{a}\right)-k_{2}^{a} F_{g}^{a}\left(\hat{t}^{a}\right) .
$$

By the definition of $\hat{t}^{a}$, we have $k_{1}^{a} f_{i}^{a}\left(y^{a}\right)>k_{2}^{a} f_{g}^{a}\left(y^{a}\right)$ for all $y^{a}<\hat{t}^{a}$. Integrating over the range $y^{a} \leq \hat{t}^{a}$ then establishes that $D_{1}^{a}>0$. Figure 2 shows $D_{1}^{a}$ as a function of $k^{b}$. From $A$ 's point of view, do-it-yourself decision-making is preferred to jury decision-making only if $k^{b}$ is sufficiently smaller than $k^{a}$. Note that $D_{1}^{a}$ is negative at $k^{b}=k^{a}$. With no conflict of preferences, jury decision-making dominates do-it-yourself decision-making because information is better.

Let $\mathrm{E}\left[C^{b}\left(Y^{a}\right)\right]$ denote juror $B$ 's unconditional expected loss when juror $A$ alone makes the decision based on his private information. Clearly, if $Y^{a}$ and $Y^{b}$ have identical conditional distributions, $\mathrm{E}\left[C^{b}\left(Y^{b}\right)\right] \leq \mathrm{E}\left[C^{b}\left(Y^{a}\right)\right]$. That is, each juror always prefers deciding by himself to letting the other juror make the decision. Let $D_{2}^{b}\left(k^{b}\right)$ be the difference between the expected loss 


\section{Figure 2}

Welfare comparison: do-it-yourself versus committee

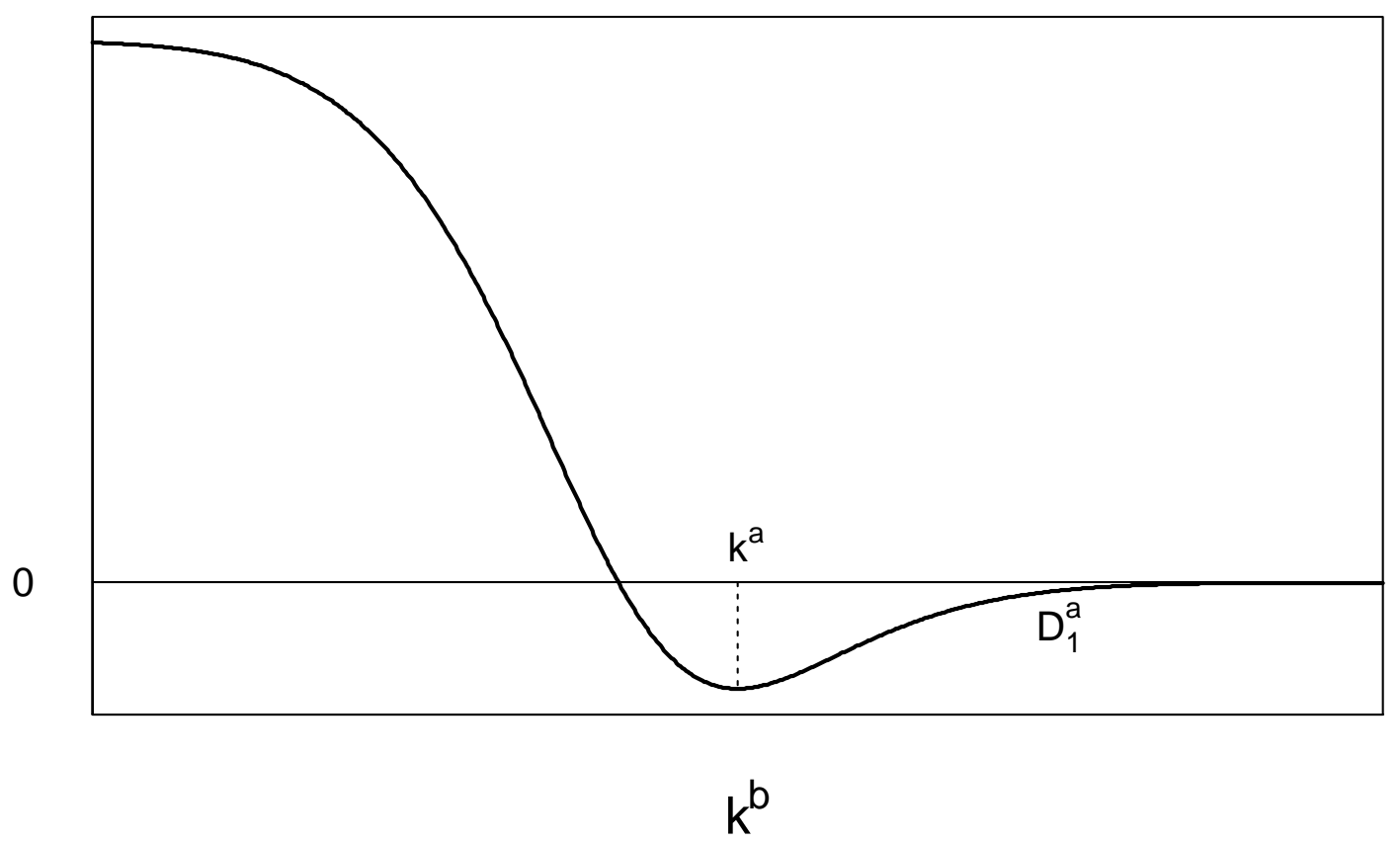

to juror $B$ under jury decision-making and his expected loss if he delegates the decision to $A$. Then, for all $k^{a}$,

$$
D_{2}^{b}=D_{1}^{b}+\mathrm{E}\left[C^{b}\left(Y^{b}\right)\right]-\mathrm{E}\left[C^{b}\left(Y^{a}\right)\right] \leq D_{1}^{b}
$$

From Figure 2 (interchanging the roles for $A$ and $B$ ), a necessary (but not sufficient) condition for $D_{2}^{b}$ to be positive is that $k^{a}<k^{b}$.

The above analysis implies that mutually agreed delegation cannot occur. For juror $B$ to prefer delegating to $A, D_{2}^{b}$ must be positive. For juror $A$ to accept the delegation, $D_{1}^{a}$ must be positive. A necessary condition for the former is that $k^{a}<k^{b}$, and a necessary condition for the latter is $k^{b}<k^{a}$. These two conditions are incompatible. The intuition of this result is clear from its derivation. For the delegation of decision-making to $A$ to be 
agreeable to both jurors, the benefits of using $B$ 's information in committee decision-making must be small to both $A$ and $B$. Since conviction can be ensured unilaterally, the benefits are small to $B$ only if $A$ is more biased toward conviction, and the benefits are small to $A$ only if $B$ is more biased toward conviction. Thus, if delegating decision-making to $A$ is acceptable to $A$, it will not be so to $B .^{8}$

We can also show that it is never a Pareto improvement for the two jurors to dissolve the committee and make decisions by taking turns. Let $D_{3}^{a}=\left(D_{1}^{a}+D_{2}^{a}\right) / 2$ be the difference in juror $A^{\prime}$ s expected loss from jury decision-making and his expected loss from taking turns. Since $k^{b}<k^{a}$ is a necessary condition both for $D_{1}^{a} \geq 0$ and for $D_{2}^{a} \geq 0$, it is also a necessary condition for $D_{3}^{a} \geq 0$. On the other hand, for juror $B$ to prefer taking turns to jury decision-making (i.e., $D_{3}^{b} \geq 0$ ), a necessary condition is $k^{b}>k^{a}$. It follows that at least one party will object to dissolving the committee and making decisions by taking turns instead. ${ }^{9}$

Welfare comparisons between jury decision-making and delegation or taking turns, do not change if conviction is reached unanimously instead of unilaterally. With unanimous conviction, the decisive vote is acquittal. For the delegation of decision-making from to $B$ to be agreeable to both jurors,

\footnotetext{
8 Side payments can result in mutually agreed delegation. If $A$ cares about both false conviction and false acquittal sufficiently more than $B$, there will exist side payments from $A$ to $B$ that the delegation of decision-making to $A$ is acceptable to both. Unfortunately, side payments involve inter-personal comparisons of expected loss, which are not meaningful in our model. Individual juror's behavior is not changed as long as the ratio of type I cost to type II cost is the same, but comparisons of expected loss depend on the level of the two costs.

9 The conclusion that it is never Pareto optimal to make decisions by taking turns may not hold when individual members must bear the cost of gathering their own information. The reason is that information is a public good in committee decision-making, and is under-provided due to the free-rider problem. For implications of the free-rider problem to committee decision-making, see Li (1999).
} 
the benefits of using $A$ 's information in committee decision-making must be small to both $A$ and $B$. Since acquittal can be ensured unilaterally, the benefits are small to $A$ only if $B$ is more biased toward acquittal, but the benefits are small to $B$ only if $A$ is more biased toward acquittal. Delegation or taking turns can never Pareto dominate jury decision-making because the gains from information sharing will outweigh the loss from strategic information manipulation for at least one juror.

\subsection{Sequential voting}

The preceding analysis of equilibrium assumes that individuals submit votes without knowing what votes others have cast. In many settings individuals in a committee express their positions one by one, so that people know the votes of those who voted before them. How does sequential voting differ from simultaneous voting?

Consider the strategy for the juror $B$. Suppose that juror $A$ votes first and adopts a two-partition strategy: convict if and only if $y^{a} \geq t^{a}$. With unilateral conviction, if $A$ votes for conviction, there is no decision for $B$ to make. If $A$ votes for acquittal, then $B$ 's vote will be decisive. In this case, $B$ knows that $Y^{b}=y^{b}$ and $y^{a}<t^{a}$. If he votes to convict, his expected loss from false conviction is $\eta k_{1}^{b} f_{i}^{b}\left(y^{b}\right) F_{i}^{a}\left(t^{a}\right)$, where $\eta$ is a normalizing factor. If

he votes to acquit, his expected loss from false acquittal is $\eta k_{2}^{b} f_{g}^{b}\left(y^{b}\right) F_{g}^{a}\left(t^{a}\right)$. Juror $B$ therefore votes to convict if and only if $y^{b} \geq t^{b}$, where $t^{b}$ satisfies

$$
\frac{f_{g}^{b}\left(t^{b}\right)}{f_{i}^{b}\left(t^{b}\right)} \frac{F_{g}^{a}\left(t^{a}\right)}{F_{i}^{a}\left(t^{a}\right)}=k^{b} .
$$

This condition is exactly the in equation (3.1). Now consider A's problem. If $A$ votes to convict, the case is decided regardless of juror $B$ 's vote. If $A$ 
votes to acquit, he expects juror $B$ to adopt a two-partition strategy with the threshold determined by the above equation. But the decision problem $A$ faces as a first-mover is exactly the same as the problem he faces when the two members vote simultaneously. Thus, the sequential voting game has the same as simultaneous voting.

There are two reasons sequential voting is equivalent to simultaneous voting in this particular setting. First, the pivotal voting argument is equally valid in both situations: the threshold of the second juror does not depend on whether he knows how the first juror has voted. Second, the first mover is unable to commit to a decision rule. Unlike, say, in an oligopoly game where the strategy is directly observable in a sequential model, the strategy in the strategic voting game is not observable even when the reports are sequentially submitted. Strategies in this game take the form of reporting some number if and only if the private signal exceeds a certain threshold. While the threshold value can be made public through various devices, the value of the signal is not public by assumption. As a result, any attempt to commit to a certain strategy is not verifiable. Because of this inability to commit, juror $A$ cannot manipulate juror $B$ 's decision rule, even though $B$ 's threshold depends on $A$ 's threshold through equation (3.1).

To see the latter point, suppose $A$ could commit to a decision rule before he observes his signal. Then, his optimal threshold $t^{a}$ would minimize the unconditional expected cost, $\mathrm{E}\left[C^{a}\left(Y^{a}, Y^{b}\right)\right]$. At $t^{a}$ equal to $t_{*}^{a}$, the equilibrium value without commitment, the derivative of expected loss with respect to $t^{a}$ is

$$
\frac{d \mathrm{E}\left[C^{a}\left(Y^{a}, Y^{b}\right)\right]}{d t^{a}}=\left[-k_{1}^{a} F_{i}^{a}\left(t_{*}^{a}\right) f_{i}^{b}\left(t_{*}^{b}\right)+k_{2}^{a} F_{g}^{a}\left(t_{*}^{a}\right) f_{g}^{b}\left(t_{*}^{b}\right)\right] \frac{d t^{b}}{d t^{a}}
$$


where the function $t^{b}\left(t^{a}\right)$ is defined according to the reaction function of $B$. Since $d t^{b} / d t^{a}<0$ and the term in brackets in (3.6) has the same sign as $k^{b}-k^{a}, d \mathrm{E}\left[C^{a}\left(Y^{a}, Y^{b}\right)\right] / d t^{a}$ has the same sign as $k^{a}-k^{b}$ at $t^{a}=t_{*}^{a}$. Thus, unless $k^{a}=k^{b}, A$ always has incentives to manipulate his threshold in order to influence the second juror's threshold, if $A$ could commit to a decision rule. In particular, if $k^{a}>k^{b}$, juror $A$ has incentives to lower his equilibrium threshold for conviction. By doing so, he raises the equilibrium threshold for juror $B$.

The above analysis implies that both jurors are better off when juror $A$ can commit to a threshold than when he is unable to commit. ${ }^{10}$ It was shown above that manipulation of thresholds by the two jurors under simultaneous voting leads to inefficiency loss for both of them relative to what can achieved through a commitment to jointly chosen thresholds. In particular, manipulation implies that the difference between the equilibrium thresholds is greater than what is implied by the difference in the preferences of the two jurors. The same sub-optimal outcome results under sequential voting when the first voter cannot commit to a threshold. But if that juror were able to commit, he would no longer face the incentive to respond to the other's threshold after observing his private signal. This improves juror $B$ 's welfare as well as juror $A$ 's. More precisely, suppose $k^{a}>k^{b}$, so that juror $A$ is more biased than juror $B$ toward acquittal. Juror $A$ would lower his conviction threshold, making $B$ better off. Juror $B$ would raise his own threshold in response, which makes $A$ better off.

10 As in oligopoly models, commitment of the first-mover to a threshold decision rule can be difficult because it is time-inconsistent. However, credible commitment may be achieved by delegating decision-making through clear procedures and rules. Our result here is consistent with the observation that a beneficial side of bureaucratic decision-making procedures is reduction in strategic manipulations of information in the committee. 


\section{Abstention}

So far abstention has not been allowed in voting. This assumption may appear innocuous. In the case of unilateral conviction, for example, allowing jurors to abstain from voting after observing their evidence will not change behavior because abstention is equivalent to voting for acquittal. However, if abstention is allowed, jurors take the abstention of others into account and equilibrium thresholds change. In this model abstention improves the quality of decision-making.

We need to specify what happens when both jurors decide to abstain. The simplest way is to specify a "default decision" when both abstain. If the default is acquittal, abstaining is still equivalent to voting for acquittal and therefore has no effect on the equilibrium. But suppose the default is conviction. Then a vote to acquit by $A$ results in conviction only if $B$ votes to convict, while abstention by $A$ results in conviction when $B$ either votes to convict or abstains. Since abstention is more likely to result in conviction than a vote for acquittal, we expect equilibrium strategies to involve two thresholds, $t_{1}^{j}<t_{2}^{j}$, such that a juror strategy is

$$
R^{j}\left(y^{j}\right)= \begin{cases}\text { "convict", } & \text { if } y^{j} \geq t_{2}^{j} \\ \text { "abstain", } & \text { if } t_{2}^{j}>y^{j} \geq t_{1}^{j} \\ \text { "acquit", } & \text { if } y^{j}<t_{1}^{j} .\end{cases}
$$

Using similar reasoning as in the proof of Proposition 3.1, we can establish that the thresholds for juror $A$ satisfy:

$$
\begin{gathered}
\frac{f_{g}^{a}\left(t_{1}^{a}\right)}{f_{i}^{a}\left(t_{1}^{a}\right) \frac{F_{g}^{b}\left(t_{2}^{b}\right)-F_{g}^{b}\left(t_{1}^{b}\right)}{F_{i}^{b}\left(t_{2}^{b}\right)-F_{i}^{b}\left(t_{1}^{b}\right)}}=k^{a}, \\
\frac{f_{g}^{a}\left(t_{2}^{a}\right)}{f_{i}^{a}\left(t_{2}^{a}\right)} \frac{F_{g}^{b}\left(t_{1}^{b}\right)}{F_{i}^{b}\left(t_{1}^{b}\right)}=k^{a} ; \\
-28-
\end{gathered}
$$


and a symmetric pair of equations holds for juror $B$. The term $\left(F_{g}^{b}\left(t_{2}^{b}\right)-\right.$ $\left.F_{g}^{b}\left(t_{1}^{b}\right)\right) /\left(F_{i}^{b}\left(t_{2}^{b}\right)-F_{i}^{b}\left(t_{1}^{b}\right)\right)$ in the first equation of (4.1) is the likelihood that $B$ abstains. In that case, $A$ can guarantee acquittal only if he votes to acquit. The term $F_{g}^{b}\left(t_{1}^{b}\right) / F_{i}^{b}\left(t_{1}^{b}\right)$ in the second equation is the likelihood that $B$ votes to acquit. In that case, $A$ can guarantee acquittal if he abstains. The monotone likelihood ratio condition implies that $\left(F_{g}^{j}\left(t_{2}^{j}\right)-F_{g}^{j}\left(t_{1}^{j}\right)\right) /\left(F_{i}^{j}\left(t_{2}^{j}\right)-\right.$ $\left.F_{i}^{j}\left(t_{1}^{j}\right)\right)>f_{g}^{j}\left(t_{1}^{j}\right) / f_{i}^{j}\left(t_{1}^{j}\right)$ and $F_{g}^{j}\left(t_{1}^{j}\right) / F_{i}^{j}\left(t_{1}^{j}\right)<f_{g}^{j}\left(t_{1}^{j}\right) / f_{i}^{j}\left(t_{1}^{j}\right)$. Then, if $t_{2}^{b}>t_{1}^{b}$, (4.1) implies that $t_{2}^{a}>t_{1}^{a}$, and vice versa. Thus, the thresholds $\left(t_{1}^{a}, t_{2}^{a}, t_{1}^{b}, t_{2}^{b}\right)$ defined by (4.1) form a Nash equilibrium.

Comparing the thresholds in the equilibrium with abstention with the equilibrium thresholds without abstention shows that allowing abstention makes committee members more "careful" in casting their votes. If the evidence is not very strong either way, a juror chooses to abstain. Standards of evidence for voting to convict or to acquit are raised so that the probability of voting either way is reduced for both members.

Proposition 4.1. If $l^{j}(\cdot) / L_{*}^{j}(\cdot)$ is monotonically increasing for each $j=a, b$, then $t_{2}^{j}>t_{*}^{j}>t_{1}^{j}$.

Proof. See the appendix.

Q.E.D.

Allowing abstention is formally identical to allowing each committee member to submit three reports instead of two. ${ }^{11}$ With no conflict of preferences, truthful revelation of private evidence is an equilibrium. Abstention

11 In the case of unilateral conviction, the two reports $r_{0}^{j}$ and $r_{1}^{j}$ for each juror $j$ satisfy the property that $S\left(r_{1}^{a}, r_{0}^{b}\right) \geq 0, S\left(r_{0}^{a}, r_{1}^{b}\right) \geq 0$, and $S\left(r_{0}^{a}, r_{0}^{b}\right)<0$. Find a report $\bar{r}^{a} \in\left(r_{0}^{a}, r_{1}^{a}\right)$ for juror $A$ such that $S\left(\bar{r}^{a}, r_{0}^{b}\right)<0$. Since $S\left(\bar{r}^{a}, r_{1}^{b}\right)>S\left(r_{0}^{a}, r_{1}^{b}\right) \geq 0$, we can find a report $\bar{r}^{b} \in\left(r_{0}^{b}, r_{1}^{b}\right)$ for juror $B$ such that $S\left(\bar{r}^{a}, \bar{r}^{b}\right) \geq 0$ and $S\left(r_{0}^{a}, \bar{r}^{b}\right)<0$. The three reports $r_{0}^{j}<\bar{r}^{j}<r_{1}^{j}$ for each juror $j$, together with the decision function $S\left(r^{a}, r^{b}\right)$, then implement the voting rule with conviction as the default decision when both abstain. 
cannot improve the quality of committee decision, because valuable evidence is thrown away. However, when preferences conflict and abstention is not allowed, members are forced to take more extreme positions in voting. This is shown in Proposition 4.1. Allowing abstention improves the expected welfare of committee members by reducing harmful strategic manipulations in the committee. Moreover, abstention allows each juror to adopt a reporting strategy involving three partitions instead of two. Finer partitioning of information improves the welfare of the committee.

To illustrate how the option to abstain improves welfare of the committee, we consider a Cournot tatonnement process that begins with the twopartition equilibrium without abstention and converges towards the threepartition equilibrium with abstention. Note that any two-partition strategy can be viewed as a three-partition strategy by adding an additional threshold for each juror appropriately. For $j=a, b$, let $\underline{y}^{j}$ and $\bar{y}^{j}$ be the lower and the upper bound of the support $Y^{j}$. If $z_{1}^{a}=\underline{y}^{a}$ and $z_{2}^{a}=t_{*}^{a}$ are juror $A^{\prime}$ s two thresholds, and $z_{1}^{b}=t_{*}^{b}$ and $z_{2}^{b}=\bar{y}^{b}$ are $B$ 's two thresholds, the voting outcome is the same as the two-partition equilibrium defined by (3.1). In each iteration of the Cournot tatonnement, the new thresholds are chosen as best responses to the previous thresholds. This process converges monotonically to the three-partition equilibrium. See Figure 3. Furthermore, expected cost for each juror falls in each iteration.

Proposition 4.2. Expected loss under the three-partition equilibrium is lower than expected loss under the two-partition equilibrium for each committee member.

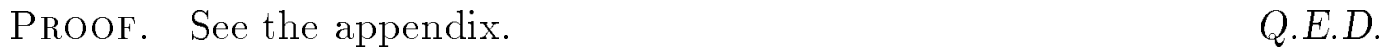




\section{Figure 3}

Cournot tatonnement: from two-partition to three-partition equilibrium
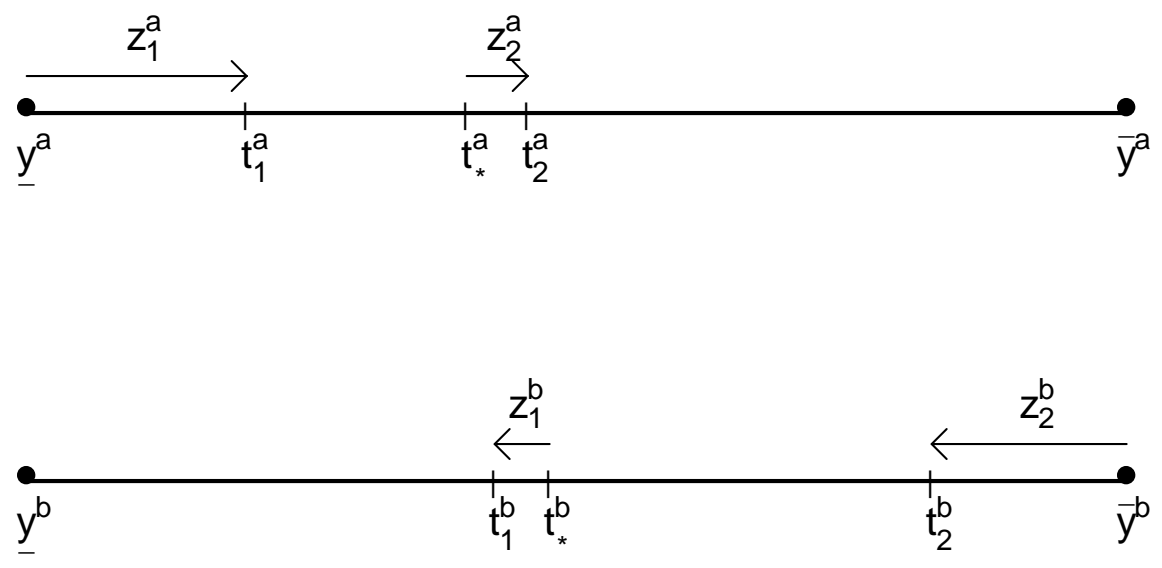

Juror $A$ is relatively biased toward conviction and $B$ toward acquittal. The proof of Proposition 4.2 shows that the reduction in the expected loss is due to two effects of abstention. One effect arises from the common interests in the committee to use whatever information available: allowing abstention provides a finer partition of information. The other effect is the increase in $A$ 's threshold of conviction and the corresponding decrease in $B$ 's threshold of acquittal. Juror $A$ becomes more reluctant to unilaterally convict and juror $B$ lowers the threshold of voting for acquittal. This strategic effect arises from the conflicting interests in the committee. It disappears when $k^{a}=k^{b}$. Thus, the option to abstain has the additional benefit of softening the positions that committees members are forced to take when abstention is disallowed.

\section{Finer Partitions}

If the option to abstain increases the effective number of partitions in the information-reporting game from two to three, are there other equilibria that 
support finer partitioning of information? The answer is yes, but differences in preferences limit how fine the partitions can be.

Fix any number $N \geq 1$ and a committee decision rule "convict if and only if $S\left(r^{a}, r^{b}\right) \geq 0$ " with a strictly increasing function $S$. We construct an equilibrium where each juror $j(j=a, b)$ uses a $(N+1)$-partition strategy, with reports $r_{0}^{j}<r_{1}^{j}<\ldots<r_{N}^{j}$, such that for each $n=0, \ldots, N, r_{n}^{j}$ is reported if $y^{j} \in\left[t_{n}^{j}, t_{n+1}^{j}\right)$, where $t_{1}^{j}, \ldots, t_{N}^{j}$ are the $N$ thresholds $\left(t_{0}^{j}=\underline{y}^{j}\right.$ and $t_{N+1}^{j}=\bar{y}^{j}$ are defined as the lower and upper bound of the support of $Y^{j}$.) The reports $r_{0}^{j}, \ldots, r_{N}^{j}$ satisfy the property (and a symmetric one by interchanging the roles of $a$ and $b)$ that for each $n=0,1, \ldots, N, S\left(r_{N-n}^{a}, r_{n}^{b}\right) \geq 0$ and $S\left(r_{N-n-1}^{a}, r_{n}^{b}\right)<0$. This is a "pivotal" condition for the reports. Unlike the equilibrium with two-partition strategies, each juror can convey the strength of his evidence by choosing different reports. A report $r_{n}^{b}$ by juror $B$ results in conviction only when juror $A$ chooses a report at least as large as $r_{N-n}^{a}$. We consider the case of unilateral conviction by assuming $S\left(r_{N}^{a}, r_{0}^{b}\right) \geq 0$ and $S\left(r_{0}^{a}, r_{N}^{b}\right) \geq 0$ : submitting a report $r_{N}^{j}$ ensures conviction regardless of the report of the other juror.

Existence of the reports that satisfy the above property can be shown by induction, similar to how Section 4 showed that abstention can be implemented by adding another report for each committee member. Moreover, if juror $A$ adopts the above reporting strategy with the $N+1$ reports $r_{0}^{a}, \ldots, r_{N}^{a}$, juror $B$ has no incentives to use reports other than $r_{0}^{b}, \ldots, r_{N}^{b}$. For example, any report $r^{b} \in\left(r_{n}^{b}, r_{n+1}^{b}\right)$ such that $S\left(r_{N-n-1}^{a}, r^{b}\right)<0$ is the same as $r_{n}^{b}$ (because both result in conviction if and only if $r^{a} \geq r_{N-n}^{a}$ ), and any $r^{b} \in\left(r_{n}^{b}, r_{n+1}^{b}\right)$ such that $S\left(r_{N-n-1}^{a}, r^{b}\right) \geq 0$ is the same as $r_{n+1}^{b}$. As in the two-partition case, many sets of reports satisfy the pivotal conditions for an $(N+1)$-partition equilibrium, but they all lead to the same equilibrium. 
For illustration, suppose each juror submits a score of 1 to 10 , and the verdict is conviction if the total score is at least 7 . Then submitting any score of 7 or above will ensure conviction unilaterally and is strategically equivalent. Each juror has therefore 7 strategically distinct reports. A report of, say, 4 leads to conviction if and only if the other juror submits a report at least as large as 3 . This decision rule induces a 7 -partition equilibrium. If the committee convicts whenever the total score is at least 15 , then submitting any score of 4 or below will ensure acquittal unilaterally. This induces another 7-partition equilibrium corresponding to the case of unanimous conviction.

Deriving conditions for the $(N+1)$-partition equilibrium is a straightforward extension of the proof of Proposition 3.1. A pivotal voting argument is also available. By construction, for each $n=1, \ldots, N$, a choice between $r_{n-1}^{a}$ and $r_{n}^{a}$ for juror $A$ is pivotal only if juror $B$ reports $r_{N-n}^{b}$ : if juror $A$ reports $r_{n-1}^{a}$ there is acquittal, and if he reports $r_{n}^{a}$ there is conviction. Juror $A$ therefore makes the choice between the two reports conditional on his evidence $Y^{a}=y^{a}$ and on juror $B^{\prime}$ s report $r^{b}=r_{N-n}^{b}$ (that is, $y^{b} \in\left[t_{N-n}^{b}, t_{N-n+1}^{b}\right)$ ). The expected loss to juror $A$ from choosing $R_{n}^{a}$ is

$$
\eta k_{1}^{a} f_{i}^{a}\left(y^{a}\right)\left(F_{i}^{b}\left(t_{N-n+1}^{b}\right)-F_{i}^{b}\left(t_{N-n}^{b}\right)\right),
$$

and from choosing $r_{n-1}^{a}$ is

$$
\eta k_{2}^{a} f_{g}^{a}\left(y^{a}\right)\left(F_{g}^{b}\left(t_{N-n+1}^{b}\right)-F_{g}^{b}\left(t_{N-n}^{b}\right)\right),
$$

where $\eta$ is a probability normalization factor under Bayesian updating. The term $F_{k}^{b}\left(t_{N-n+1}^{b}\right)-F_{k}^{b}\left(t_{N-n}^{b}\right)(k=i, g)$ is the probability that $B$ 's evidence 
lies in the interval that allows $A$ to be pivotal. Thus, reporting $r_{n}^{a}$ instead of $r_{n-1}^{a}$ is optimal if and only if $y^{a} \geq t_{n}^{a}$ where the threshold $t_{n}^{a}$ satisfies

$$
\frac{f_{g}^{a}\left(t_{n}^{a}\right)}{f_{i}^{a}\left(t_{n}^{a}\right)} \frac{F_{g}^{b}\left(t_{N-n+1}^{b}\right)-F_{g}^{b}\left(t_{N-n}^{b}\right)}{F_{i}^{b}\left(t_{N-n+1}^{b}\right)-F_{i}^{b}\left(t_{N-n}^{b}\right)}=k^{a} .
$$

From the monotone likelihood ratio property, we can establish that $\left[F_{g}^{j}(u)-\right.$ $\left.F_{g}^{j}(v)\right] /\left[F_{i}^{j}(u)-F_{i}^{j}(v)\right]$ is increasing in both $u$ and $v$ for all $u>v .{ }^{12}$ Since the above argument holds for $n=1, \ldots, N$, the thresholds defined by equations (5.1) satisfy $t_{1}^{a}<\ldots<t_{N}^{a}$. Thus, the pivotal voting argument proves that if juror $B$ uses a reporting strategy with thresholds $t_{1}^{b}<\ldots<t_{N}^{b}$, the strategy defined by (5.1) is optimal for juror $A$.

To conclude, the thresholds for an equilibrium with a $(N+1)$-partition strategies are described by the $N$ equations in (5.1), plus a symmetric set of $N$ equations for juror $B$. The conditions (3.1) for the two-partition equilibrium and conditions (4.1) for the three-partition equilibrium are special cases of (5.1). If jurors have identical preferences, the partitions get finer and finer as $N$ increases. The solution converges to that implied by the Neyman-Pearson lemma, and full information revelation occurs. However, conflicts from differences in preferences place an upper bound on how fine the partitions can be in equilibrium. ${ }^{13}$

12 The derivative of this ratio with respect to $u$ has the same sign as $f_{g}^{j}(u)\left[F_{i}^{j}(u)-\right.$ $\left.F_{i}^{j}(v)\right]-f_{i}^{j}(u)\left[F_{g}^{j}(u)-F_{g}^{j}(v)\right]$. By the monotone likelihood ratio property, $f_{g}^{j}(u) f_{i}^{j}(y) \geq$ $f_{i}^{j}(u) f_{g}^{j}(y)$ for all $y \leq u$. Integrating over $y$ from $v$ to $u$ gives $f_{g}^{j}(u)\left[F_{i}^{j}(u)-F_{i}^{j}(v)\right] \geq$ $f_{i}^{j}(u)\left[F_{g}^{j}(u)-F_{g}^{j}(v)\right]$. Monotonicity in $v$ can be proved in a similar manner.

13 For any given $N$, the equations (5.1) define a Nash equilibrium. Existence of the equilibrium for an arbitrary $N$ can be guaranteed in the same way as in the two-partition case. This existence result does not contradict the result in the following proposition that the number of partitions is bounded on any strict subset of the support of evidence. For a larger $N$, more of the corresponding equilibrium thresholds are squeezed into the two ends of the support. 
Proposition 5.1. If $k^{a} \neq k^{b}$, then for any interval $\left[y_{\min }^{j}, y_{\max }^{j}\right]$ in the support of $Y^{j}$ there is an $\epsilon>0$, such that for any equilibrium thresholds $t_{n}^{j}, t_{n-1}^{j} \in\left[y_{\min }^{j}, y_{\max }^{j}\right], t_{n}^{j}-t_{n-1}^{j}>\epsilon$.

Proof. The monotone likelihood ratio property implies that, for all $u>v$,

$$
l^{j}(u)>\left(F_{g}^{j}(u)-F_{g}^{j}(v)\right) /\left(F_{i}^{j}(u)-F_{i}^{j}(v)\right)>l^{j}(v),
$$

where $l^{j}(\cdot)=f_{g}^{j}(\cdot)=f_{i}^{j}(\cdot)$. From this result and from the equilibrium conditions for $t_{n-1}^{a}$ and for $t_{N+1-n}^{b}$, we have

$$
\begin{gathered}
l^{a}\left(t_{n-1}^{a}\right) l^{b}\left(t_{N+1-n}^{b}\right)<k^{a}, \\
l^{b}\left(t_{N+1-n}^{b}\right) l^{a}\left(t_{n}^{a}\right)>k^{b} .
\end{gathered}
$$

Adding these inequalities:

$$
l^{b}\left(t_{N+1-n}^{b}\right) l^{a \prime}(\zeta)\left(t_{n}^{a}-t_{n-1}^{a}\right)>k^{b}-k^{a},
$$

where $\zeta$ is between $t_{n}^{a}$ and $t_{n-1}^{a}$. Similar manipulations using the equations for $t_{n}^{a}$ and for $t_{N+1-n}^{b}$ yield

$$
l^{b}\left(t_{N+1-n}^{b}\right) l^{a \prime}(\zeta)\left(t_{n}^{a}-t_{n-1}^{a}\right)>k^{a}-k^{b} .
$$

Therefore

$$
t_{n}^{a}-t_{n-1}^{a}>\frac{\left|k^{a}-k^{b}\right|}{l^{b}\left(t_{N+1-n}^{b}\right) l^{a \prime}(\zeta)} .
$$

If $t_{n}^{a}-t_{n-1}^{a} \leq \epsilon$, then since $\epsilon$ can be arbitrarily small and since $l^{b}(\cdot)$ and $l^{a \prime}(\cdot)$ are both bounded over any fixed interval, the above inequality contradicts $k^{a} \neq k^{b}$.

Q.E.D. 


\section{Figure 4}

Conflicts and bound on fineness of partitions

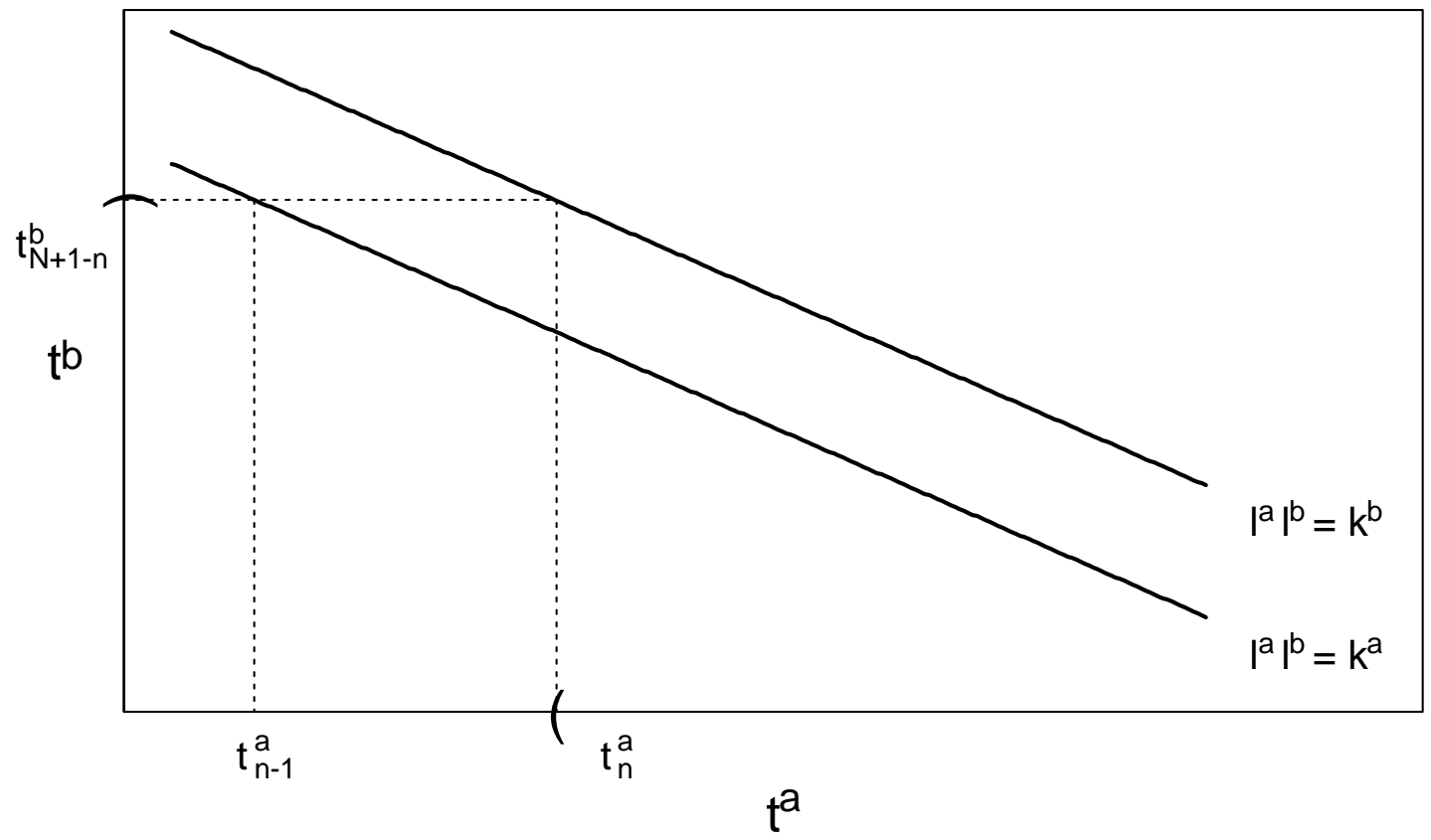

Figure 4 illustrates the argument of Proposition 5.1 for the case of $k^{b}>$ $k^{a}$. The monotone likelihood ratio property imposes bounds on the relevant thresholds as represented by the two inequalities (5.2). Since $k^{b}>k^{a}$, the curve $l^{a} l^{b}=k^{b}$ is to the right of $l^{a} l^{b}=k^{a}$ in Figure 4. For any fixed threshold $t_{n-1}^{a}$ of juror $A$, the corresponding threshold $t_{N+1-n}^{b}$ of $B$ is bounded from above through the first inequality of (5.2). Once $t_{N+1-n}^{b}$ is bounded from above, the corresponding threshold $t_{n}^{a}$ of $A$ is bounded from below through the second inequality of (5.2). Thus, the distance between $t_{n-1}^{a}$ and $t_{n}^{a}$ is bounded from below. Proposition 5.1 implies that the number of partitions in an equilibrium strategy of juror $A$ is at most $\left(y_{\max }^{a}-y_{\min }^{a}\right) / \epsilon$ on the interval $\left[y_{\min }^{a}, y_{\max }^{a}\right]$. Figure 4 makes explicit that the upper bound on the fineness of equilibrium partitions for a given interval depends negatively on 
the difference in preferences, $\left|k^{a}-k^{b}\right|$. If the two jurors have a greater difference in preferences, the two curves in Figure 4 are further apart, and the lower bound on the distance between adjacent thresholds for each juror becomes larger. Great conflicts within the committee make fine partitions impossible. ${ }^{14}$

\section{Decision Rules and Decision-making}

In a two-partition equilibrium, there are essentially just two decision rules for a committee of two members, unilateral conviction and unanimous conviction. It might seem that requiring two votes for conviction instead of one is a more "stringent" standard of proof. But this is only true when jurors cast their votes without regard to the voting rule. Since each juror cares only about the final verdict rather than his own vote, he votes to convict less cautiously when unanimity is required, knowing that the other juror may have information that will lead to a vote against conviction. On the other hand, if one vote is sufficient for a guilty verdict, each juror is more cautious in casting a vote to convict, knowing that such a vote would have a decisive effect regardless of the other juror's information. More precisely, the monotone likelihood ratio property implies that for each $j=a, b, L_{*}^{j}(\cdot) \leq 1$ and $L_{* *}^{j}(\cdot) \geq 1$. It then follows from Proposition 3.1 that $t_{*}^{j} \geq t_{* *}^{j}$. Thus, both jurors set a lower standard of conviction when the decision rule is changed from unilateral conviction to unanimous conviction. ${ }^{15}$ This aspect of comparison

14 Crawford and Sobel (1982) establish a similar result in the context of an information reporting game with one sender and one receiver.

15 This comparison of decision of decision rules complements the works of Sah and Stiglitz $(1986$; 1988), who consider committees without the strategic manipulations that arise from conflicting interests. 


\section{Figure 5}

Conflicts and personal preference over decision rules

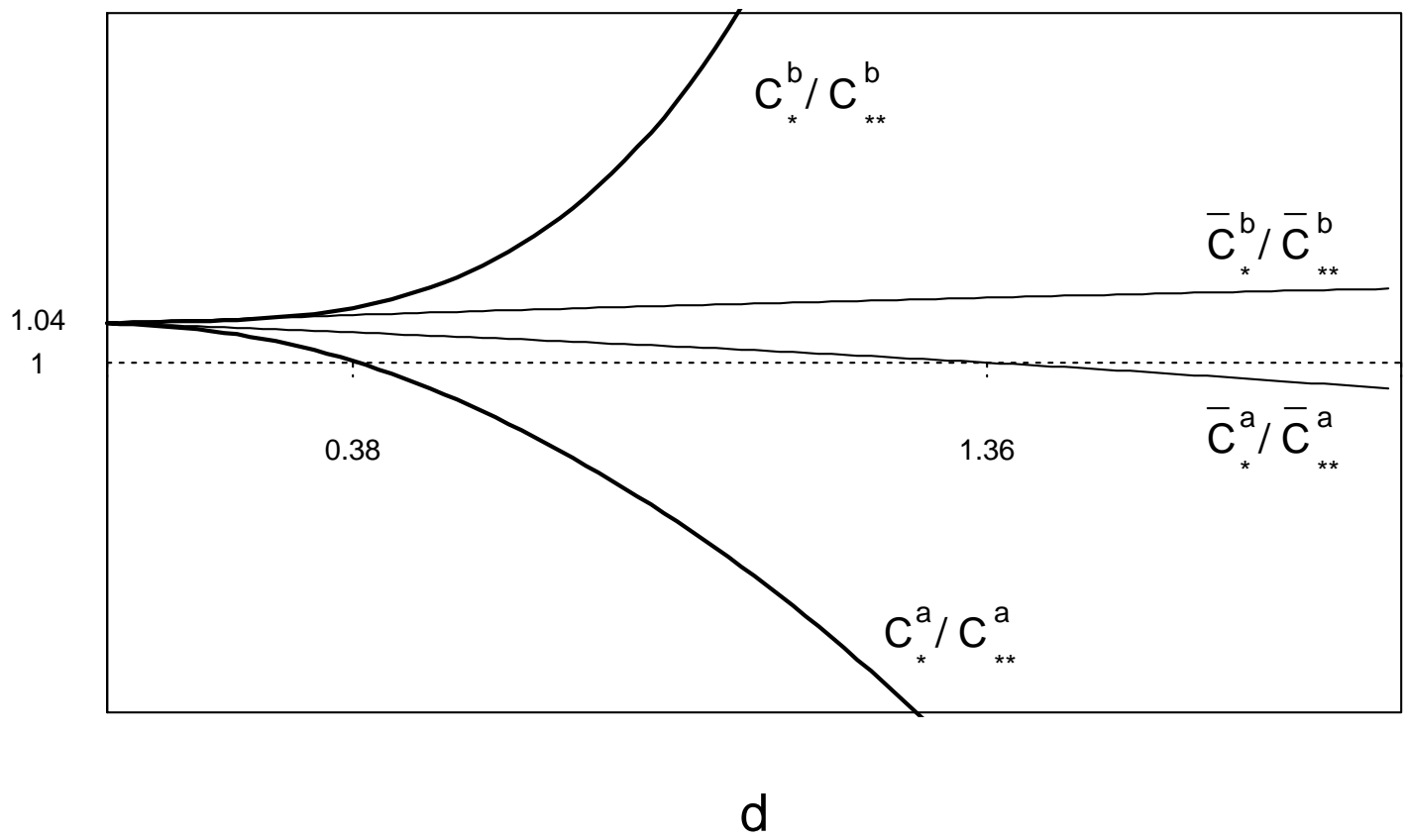

of decision rules illustrates the common interests in sharing information in committee decision-making.

The extent of conflicts in the committee affects members' preference over decision rules. When the two jurors have identical interests, they agree on which decision rule should be used in committee decision-making. By continuity, small differences in preference do not generate disagreement about the ex ante choice of decision rule. However, as conflicts increase in the committee, strategic manipulations of information amplify the differences in personal preference over decision rules. For a numerical example, let $F_{i} \sim N(0,1)$ and $F_{g} \sim N(1,1)$ be the common distribution functions, conditional on innocence and on guilt. Then, if the common preference $k$ exceeds 1 so that both jurors are relatively biased toward acquittal, unanimous con- 
viction is preferred to unilateral conviction. Now, consider how an individual juror's preference over the decision rule changes in the following comparative statics exercise. Let $k_{1}^{a}=k-d, k_{1}^{b}=k+d$, and $k_{2}^{a}=k_{2}^{b}=1$. As $d$ increases from 0 to $k, k^{a}$ decreases and $k^{b}$ increases. To examine the role of equilibrium manipulations of information, define a "cooperative" threshold $\bar{t}_{*}$ under unilateral conviction that satisfies $l\left(\bar{t}_{*}\right) L_{*}\left(\bar{t}_{*}\right)=k$. By construction, $\bar{t}_{*}$ minimizes the equally-weighted sum of expected cost to the two jurors under unilateral conviction, regardless of the extent of conflicts $d$. Similarly, define $\bar{t}_{* *}$ under unanimous conviction that satisfies $l\left(\bar{t}_{* *}\right) L_{* *}\left(\bar{t}_{* *}\right)=k$. Figure 5 illustrates how each juror $j$ 's preference over plurality changes with $d$, by plotting the ratio of his expected cost under unilateral conviction to the cost under unanimous conviction. With "cooperative" thresholds, juror $B$ 's preference for unanimous conviction becomes stronger as he becomes more biased toward acquittal (i.e., $\bar{C}_{*}^{b} / \bar{C}_{* *}^{b}$ increases with $d$ ). Juror $A$ initially shares $B$ 's preference, as shown by $\bar{C}_{*}^{a} / \bar{C}_{* *}^{a}$, but switches his preference to unilateral conviction as he becomes more concerned with false acquittal. In the numerical example shown (where $k=2$ ), this happens around $d=1.36$. In contrast, equilibrium manipulations of information arising from increasing conflicts between $A$ and $B$ in the non-cooperative game imply a larger difference in personal preference over decision rule. Figure 5 also plots the ratio of each juror $j$ 's equilibrium expected cost $C_{*}^{j}$ under unilateral conviction to the cost $C_{* *}^{j}$ under unanimous conviction. As with cooperative decision-making, the difference between $C_{*}^{b} / C_{* *}^{b}$ and $C_{*}^{a} / C_{* *}^{a}$ becomes greater as $d$ increases, but the divergence goes much faster. Juror $A$ switches his preferred decision rule from unanimous conviction to unilateral conviction around $d=0.38$, but would prefer unanimous conviction if standards were set cooperatively. 


\section{Conclusion}

Committee members' incentives to manipulate private information to tilt decisions toward their personally preferred outcome imply that information cannot be efficiently aggregated by committees. Perhaps this is the basis for the old joke: "Ques. How do committees make decisions? Ans. Badly." Nonetheless, committees are used to make many business and other decisions. We have illuminated some of the reasons for their continued use and survival. True, self interest and strategic considerations make information pooling in committees imperfect, but that is relative to some unattainable ideal. Strategic noise introduced by attempted manipulations of the outcome still leads to better decisions for all members together than if one of them acted as "dictator" and made the decision without benefit of other, albeit strategically manipulated, information. Decisions are better in the sense that not all members would prefer ex ante to dissolve the committee and randomly select a "dictator" among them to make less informed decisions.

The reason is that viable committees must share some common goals, even though individual members might weigh outcomes somewhat differently. And members certainly want to gain the statistical advantages of information sharing and sharpening the signal to noise ratio. What makes the process work is that the committee rules and procedures are themselves chosen to temper and control strategic misrepresentations and filter the data in the best way possible, given self-interested behavior. Procedures are adopted that coarsen the reporting of information and put a natural limit on feasible manipulations. They control conflict in an acceptable way. The greater the differences of a priori opinion among members, the coarser the rules must be to control conflict. The quality of committee decisions necessarily 
declines with the degree of conflict. Yet poor as committee decisions might be when conflicts are reasonably large, they still might be better than what one person could achieve for the combined interests of the group as a whole from unilateral, and less-informed, decisions.

The two-partition voting mechanism studied in detail here is a very clear analytical representation of these ideas. In the statistical decision problem from which it is constructed, all sample information is perfectly aggregated into a "score." Minimizing the loss function sets a critical score. If the sample score exceeds the threshold, the object is put into one category, and if it falls short of the threshold it is put in the other category. Voting in a committee is a cruder kind of scoring system, but a scoring system nonetheless. Each person sets his own critical standard endogenously. The object is placed into one category or another by the committee depending on the proportion of members whose sample information place it above or below their own strategically determined personal thresholds.

Some classifications that would be chosen by a hypothetical perfect aggregation scheme cannot occur in a committee. This inefficiency cannot be eliminated unless there is no conflict. Personal thresholds are chosen to "undo" the presumed biases and preferences of other members, but not by enough to completely nullify the information of others. For instance, members defer to those who have more informed sample information-members who have greater expertise and who are drawing their data out of probability distributions with greater precision - in the sense that the better informed members are decisive more often.

While there are few general analytical results on how voting thresholdssimple majority, super-majority, or unanimity - affect the quality of committee decisions, the analysis illuminates some of the economic considerations 
involved in these debates. It is interesting that though requiring unanimity for conviction makes each member pivotal for conviction, self-interest and strategy makes them less cautious in voting to convict because others are more likely to have information against conviction. On the other hand, requiring unanimity for acquittal makes voters more cautious in voting for conviction. These are precisely the reasons why Condorcet's Theorem fails when strategic considerations play a role in voting (Austin-Smith and Banks, 1996; Feddersen and Pessendorfer, 1998). Strictly speaking in our model, each issue coming before the committee would have an optimal partition and an optimal committee cutoff score on votes. These could be simulated, as illustrated in the previous section. We have chosen not to pursue this line because our model is not sufficiently well structured for that kind of analysis. Committee rules are chosen to achieve a certain kind of durability to a broad variety of issues that come before it. But the nature of preferences, voting rules, incentives to collect information and even what issues are likely to come up before the committee (Li, 1999), the presentation of arguments and rhetoric in committee deliberations (Posner, 1998; Dewatripont and Tirole, 1999), intertemporal vote trading in future deliberations for ongoing committees are all likely to be important for understanding these things. This model is too crude to incorporate such things.

In conclusion, voting is said to be an inferior allocation mechanism because it does not allow the intensity of one's preferences to be expressed in the final tally. And so it is for purely private decisions in which information and tastes of others are not directly germane. But in decisions where social gains possibly arise from the pooling information, the intensity of differences in preferences leads to discordance among members that causes trouble. Various kinds of voting procedures bound the expression of intensity and discor- 
dance among voters and lead to better informed decisions. Perhaps this is the main lesson in this paper.

\section{Appendix}

\section{A.1. Proof of Proposition 4.1}

Suppose $t_{*}^{a} \geq t_{2}^{a}$. Then, using the second equation in (4.1) and the first equation in (3.1), we have $t_{1}^{b} \geq t_{*}^{b}$. From the monotonicity of $l^{j}(\cdot) / L_{*}^{j}(\cdot)$, we get

$$
\frac{l^{b}\left(t_{1}^{b}\right)}{L_{*}^{b}\left(t_{1}^{b}\right)} \frac{l^{a}\left(t_{*}^{a}\right)}{L_{*}^{a}\left(t_{*}^{a}\right)} \geq \frac{l^{b}\left(t_{*}^{b}\right)}{l^{b}\left(t_{*}^{b}\right)} \frac{l^{a}\left(t_{2}^{a}\right)}{L_{*}^{a}\left(t_{2}^{a}\right)} .
$$

Cross multiplying and using (3.1) and (4.1) again, we get $l^{b}\left(t_{1}^{b}\right) L_{*}^{a}\left(t_{2}^{a}\right) \geq k^{b}$. The condition for the threshold $t_{1}^{b}$ is described by the equation

$$
l^{b}\left(t_{1}^{b}\right) \frac{F_{g}^{a}\left(t_{2}^{a}\right)-F_{g}^{a}\left(t_{1}^{a}\right)}{F_{i}^{a}\left(t_{2}^{a}\right)-F_{i}^{a}\left(t_{1}^{a}\right)}=k^{b}
$$

Thus, $L_{*}^{a}\left(t_{2}^{a}\right) \geq\left[F_{g}^{a}\left(t_{2}^{a}\right)-F_{g}^{a}\left(t_{1}^{a}\right)\right] /\left[F_{i}^{a}\left(t_{2}^{a}\right)-F_{i}^{a}\left(t_{1}^{a}\right)\right]$, which contradicts the monotone likelihood ratio property.

Suppose $t_{*}^{a} \leq t_{1}^{a}$. In the equilibrium without abstention, $l^{b}\left(t_{*}^{b}\right) L_{*}^{a}\left(t_{*}^{a}\right)=$ $k^{b}$. In the equilibrium with abstention, $l^{b}\left(t_{2}^{b}\right) L_{*}^{a}\left(t_{1}^{a}\right)=k^{b}$. These two conditions imply that $t_{*}^{b} \geq t_{2}^{b}$. We can then follow the same method as above to derive a contradiction.

Q.E.D.

\section{A.2. Proof of Proposition 4.2}

The proof proceeds in two steps. Step 1 shows that the two-partition equilibrium converges monotonically to the three-partition equilibrium in a Cournot 
tatonnement process. Step 2 shows that expected loss for each juror falls in each iteration of the tatonnement.

Step 1: The equilibrium conditions for the thresholds of juror $A$ specified in (4.1) can be used to define the reaction functions $z_{1}^{a}=g_{1}\left(z_{1}^{b}\right)$ and $z_{2}^{a}=$ $g_{2}\left(z_{1}^{b}, z_{2}^{b}\right)$. The reaction functions for juror $B$ can be specified analogously. Note that all the reaction functions are strictly decreasing in their arguments. If we define $x=\left(z_{1}^{a}, z_{2}^{a},-z_{1}^{b}-z_{2}^{b}\right)$ and let $h: \mathbb{R}^{4} \rightarrow \mathbb{R}^{4}$ be the reaction function in the redefined variables, then $h(x)$ is monotonic increasing in $x$. The Cournot tatonnement is specified by the process $x(t)=h(x(t-1))$. The initial thresholds are specified near the two-partition equilibrium, $x(0)=$ $\left(\underline{y}^{a}, t_{*}^{a}+\epsilon,-t_{*}^{b},-\bar{y}^{b}\right)$, where $\epsilon>0$ is arbitrarily small. An induction argument establishes that $x(t)$ increases monotonically. Suppose $x(t) \geq x(t-1)$. Then, because the reaction function $h(\cdot)$ is monotonic,

$$
x(t+1)=h(x(t)) \geq h(x(t-1))=x(t) .
$$

Furthermore, using the conditions for the two-partition equilibrium, it can be verified that $x(1)=h(x(0)) \geq x(0)$, and the induction argument is complete. A bounded and monotonic sequence converges to a limit point $\hat{x}$. By the continuity of the payoff functions, this point must also be an equilibrium point, $\hat{x}=h(\hat{x})$. To see this, note that $C^{j}\left(x^{j}(t), x^{-j}(t-1)\right) \leq C^{j}\left(x^{j}, x^{-j}(t-\right.$ 1)) for all $x^{j}(j=a, b)$ because $x(t)$ is the best response to $x(t-1)$. Since $C^{j}$ is continuous in $x^{j}$ and $x(t) \rightarrow \hat{x}$, we have $C^{j}\left(\hat{x}^{j}, \hat{x}^{-j}\right) \leq C^{j}\left(x^{j}, \hat{x}^{-j}\right)$ for all $x^{j}$. Therefore $\hat{x}$ is indeed a three-partition equilibrium point.

Step 2: Let the expected loss to juror $j$ be

$$
\begin{aligned}
C\left(z^{a}, z^{b}, k^{j}\right)= & \left.k^{j}\left[1-F_{i}^{a}\left(z_{2}^{a}\right)+\left(F_{i}^{a}\left(z_{2}^{a}\right)-F_{i}^{a}\left(z_{1}^{a}\right)\right)\left(1-F_{i}^{b}\left(z_{1}^{b}\right)\right)+F_{i}^{a}\left(z_{2}^{b}\right)\right)\right] \\
& +\left[\left(F_{g}^{a}\left(z_{2}^{a}\right)-F_{g}^{a}\left(z_{1}^{a}\right)\right) F_{g}^{b}\left(z_{1}^{b}\right)+F_{g}^{a}\left(z_{1}^{a}\right) F_{g}^{b}\left(z_{g}^{b}\right)\right] .
\end{aligned}
$$


Without loss of generality, assume $k^{a} \geq k^{b}$. The change in cost for juror $A$ between two successive iterations is

$$
\begin{aligned}
& C\left(z^{a}(t+1), z^{b}(t+1), k^{a}\right)-C\left(z^{a}(t), z^{b}(t), k^{a}\right) \\
= & {\left[C\left(z^{a}(t+1), z^{b}(t+1), k^{a}\right)-C\left(z^{a}(t), z^{b}(t+1), k^{a}\right)\right] } \\
& +\left[C\left(z^{a}(t), z^{b}(t+1), k^{a}\right)-C\left(z^{a}(t), z^{b}(t), k^{a}\right)\right] \\
\leq & \mathbf{D}_{z^{a}} C\left(z^{a}(t+1), z^{b}(t+1), k^{a}\right)\left(z^{a}(t+1)-z^{a}(t)\right) \\
& +\mathbf{D}_{z^{b}} C\left(z^{a}(t), z^{b}(t+1), k^{a}\right)\left(z^{b}(t+1)-z^{b}(t)\right),
\end{aligned}
$$

where $\mathbf{D}_{z^{a}} C$ and $\mathbf{D}_{z^{b}}$ are the gradient vectors of $C$ with respect to $z^{a}$ and $z^{b}$. The inequality above follows from the convexity of the cost function. Because convergence is monotonic, we have $z^{a}(t+2) \geq z^{a}(t+1)$. Convexity of the cost function in $z^{a}$ and the fact that $z^{a}(t+2)$ is a best response to $z^{b}(t+1)$ then imply

$$
\mathbf{D}_{z^{a}} C\left(z^{a}(t+1), z^{b}(t+1), k^{a}\right) \leq \mathbf{D}_{z^{a}} C\left(z^{a}(t+2), z^{b}(t+1), k^{a}\right)=0 .
$$

Furthermore, since $\mathbf{D}_{z^{b}} C$ is decreasing in $k$, and since $k^{a} \leq k^{b}$, we have

$$
\mathbf{D}_{z^{b}} C\left(z^{a}(t), z^{b}(t+1), k^{a}\right) \geq \mathbf{D}_{z^{b}} C\left(z^{a}(t), z^{b}(t+1), k^{b}\right)=0 .
$$

Finally, the monotonicity of the convergence process implies that $z^{a}(t+1)-$ $z^{a}(t) \geq 0$ and $z^{b}(t+1)-z^{b}(t) \leq 0$. Thus the change in cost for juror $A$ is negative.

For juror $B$, we follow a different decomposition to get

$$
\begin{aligned}
& C\left(z^{a}(t+1), z^{b}(t+1), k^{b}\right)-C\left(z^{a}(t), z^{b}(t), k^{b}\right) \\
= & {\left[C\left(z^{a}(t+1), z^{b}(t+1), k^{b}\right)-C\left(z^{a}(t+1), z^{b}(t), k^{b}\right)\right] } \\
& +\left[C\left(z^{a}(t+1), z^{b}(t), k^{b}\right)-C\left(z^{a}(t), z^{b}(t), k^{b}\right)\right] \\
\leq & \mathbf{D}_{z^{b}} C\left(z^{a}(t+1), z^{b}(t+1), k^{b}\right)\left(z^{b}(t+1)-z^{b}(t)\right) \\
& +\mathbf{D}_{z^{a}} C\left(z^{a}(t+1), z^{b}(t), k^{b}\right)\left(z^{a}(t+1)-z^{a}(t)\right),
\end{aligned}
$$


Because convergence is monotonic, we have $z^{b}(t+2) \leq z^{b}(t+1)$. Convexity

of the cost function in $z^{b}$ and the fact that $z^{b}(t+2)$ is a best response to $z^{a}(t+1)$ then imply

$$
\mathbf{D}_{z^{b}} C\left(z^{a}(t+1), z^{b}(t+1), k^{a}\right) \geq \mathbf{D}_{z^{a}} C\left(z^{a}(t+1), z^{b}(t+2), k^{a}\right)=0 .
$$

Furthermore, since $\mathbf{D}_{z^{a}} C$ is decreasing in $k$, and since $k^{a} \leq k^{b}$, we have

$$
\mathbf{D}_{z^{a}} C\left(z^{a}(t+1), z^{b}(t), k^{b}\right) \leq \mathbf{D}_{z^{a}} C\left(z^{a}(t+1), z^{b}(t), k^{a}\right)=0 .
$$

Finally, since $z^{a}(t+1)-z^{a}(t) \geq 0$ and $z^{b}(t+1)-z^{b}(t) \leq 0$, the change in cost for juror $B$ is also negative.

Q.E.D.

\section{References}

Austen-Smith, David. "Information Transmission in Debate." American Journal of Political Science 34 (February 1990): 124-152.

Austen-Smith, David, and Banks, Jeffrey S. "Information Aggregation, Rationality and the Condorcet Jury Theorem." American Political Science Review 90 (January 1996): 34-45.

Condorcet, M.J.A.N. de Caritat. An Essay on the Application of Analysis to the Probability of Decisions Rendered by a Plurality of Votes, 1875. Abridged and translated in Iain McLean and Arnold B. Urken, eds., Classics of Social Choice. Ann Arbor: University of Michigan Press, 1995.

Crawford, Vincent P., and Sobel, Joel. "Strategic Information Transmission." Econometrica 50 (November 1982): 1431-1451.

DeGroot, Morris H. Optimal Statistical Decisions. New York: McGraw Hill, 1970.

Dewatripont, Mathias, and Tirole, Jean. "Advocates." Journal of Political Economy 107 (February 1999): 1-39.

Dixit, Avinash. "Comparative Statics for Oligopoly." International Economic Review 27 (February 1986): 107-122. 
Edwards, A.W.F. Likelihood expanded edition. Baltimore: Johns Hopkins University Press, 1992.

Feddersen, Timothy J., and Pesendorfer, Wolfgang. "The Swing Voter's Curse." American Economic Review 86 (June 1996): 408-424.

Feddersen, Timothy J., and Pesendorfer, Wolfgang. "Voting Behavior and Information Aggregation in Elections with Private Information." Econometrica 65 (September 1997): 1029-1058.

Feddersen, Timothy J., and Pesendorfer, Wolfgang. "Convicting the Innocent: The Inferiority of Unanimous Jury Verdicts under Strategic Voting." American Political Science Review 92 (March 1998): 23-35.

Gibbard, A. "Manipulation of Voting Schemes: A General Result." Econometrica 41 (1973): 587-602.

Klevorick, Alvin K., Rothschild, Michael, and Winship, Christopher. "Information Processing and Jury Decisionmaking." Journal of Public Economics 23 (1984): 245-278.

Li, Hao. "A Theory of Conservatism." Unpublished manuscript, University of Hong Kong, 1999.

Posner, Richard A. "An Economic Approach to the Law of Evidence." Unpublished manuscript, University of Chicago, 1998.

Sah, Raaj Kumar, and Stiglitz, Joseph E. "The Architecture of Economic Systems: Hierarchies and Polyarchies." American Economic Review 76 (September 1986): 716-727.

Sah, Raaj Kumar, and Stiglitz, Joseph E. "Committees, Hierarchies and Polyarchies." Economic Journal 98 (June 1988): 451-470.

Satterthwaite, M. "Strategy-Proofness and Arrow's Conditions: Existence and Corresponding Theorems for Voting Procedures and Social Welfare Functions." Journal of Economic Theory 10 (1975): 187-217. 